\title{
Varietätenwandel im Kontakt. Die Entwicklung des Sprachgebrauchs deutschsprachiger Minderheiten im bundesdeutschen Kontext
}

\begin{abstract}
Die Fragestellung der Jahrestagung „Das Deutsch der Migranten“ wird am Beispiel des gesprochenen Deutsch der russischsprachigen Zuwanderer der ersten Generation (Einwanderungsgeneration) untersucht. Hervorgehoben werden auffällige Aspekte einer Langzeitstudie zum gruppenexternen und gruppeninternen Sprachgebrauch dieser Sprechergruppe im bundesdeutschen Kontext. Durch die besondere Migrationskonstellation stellen sich Sprachwandelprozesse ein, die als typisch für die Einwanderungsgeneration dieser Migrantengruppe zu betrachten sind. Die Ergebnisse beziehen sich auf die im Institut für Deutsche Sprache seit den frühen 1990er Jahren und im letzten Jahr erneut durchgeführten Sprachaufnahmen zum Sprachgebrauch der russlanddeutschen Minderheit aus der ehemaligen Sowjetunion.
\end{abstract}

\section{Einleitung ${ }^{1}$}

Unter der „Sprache der Migranten“ sind allgemein Varietäten zu verstehen, die aus linguistischer Sicht mehr oder weniger auffällige Besonderheiten im Vergleich zu den üblichen Varietäten der einheimischen bzw. autochthonen, dauernd ansässigen Bevölkerung des Einwanderungslandes aufweisen. Bei der ersten Generation der Migranten (der Einwanderungsgeneration) resultieren diese Besonderheiten in erster Linie aus dem Sprachkontakt zwischen der Herkunftssprache der Migranten und der Landessprache im Zuwanderungsland nach der Migration. Die bekanntesten Migrantensprachen sind die verschiedenen Varietäten der „Gastarbeiter“ in Europa, die im Spiegel dieser Kontaktprozesse zu sehen sind (z.B. das „Gastarbeiterdeutsch“, vgl. Keim 1982). Es sind die entsprechenden Herkunftssprachen, die neben anderen Faktoren die spezifischen Formen der Migrantensprachen der Einwanderungsgeneration bestimmen.

Andere Typen des Kontakts und Wandels infolge von Migration liegen vor, wenn verschiedene Varietäten einer Sprache in Kontakt kommen (Varietätenkontakt). Diese Konstellation ist nicht selten und ist für verschiedene

\footnotetext{
1 Verschiedene Versionen dieses Beitrags wurden im Institut für Deutsche Sprache (Mannheim) diskutiert. Mein herzlicher Dank gilt Arnulf Deppermann, Ludwig M. Eichinger, Elena Frick, Stefan Kleiner und Ralf Knöbl.
} 
Regionen der Welt beschrieben worden. Dabei kann es zu sehr unterschiedlichen Ausprägungen des Varietätenkontakts kommen. So können zwei Standardvarietäten einer Sprache (wie z.B. das britische und kanadische bzw. das amerikanische Englisch) in Kontakt kommen oder es können verschiedene Dialekte einer Sprache aufeinander treffen (Chambers 1992; Trudgill 1983, 1986; Kerswill 1994). Wichtig ist jedoch nicht nur die Frage, welche Varietäten einer Sprache miteinander in Kontakt kommen, sondern insbesondere auch die Frage der Unterschiede in den sprachlichen Repertoires der Zugewanderten und der autochthonen Bevölkerung. Eine Typologisierung der verschiedenen Konstellationen mit Berücksichtigung des Faktors Varietätenrepertoire hat Auer (2007) vorgenommen. Die uns hier interessierende Gruppe der deutschsprachigen Minderheiten aus Osteuropa fasst er als einen spezifischen Typ zusammen und beschreibt ihn wie folgt:

[...] The immigrants speak only dialect and the receiving area has a more complex repertoire including a different dialect and an overarching standard. A case in point is remigration of extraterritorial groups of speakers ,under the roof $f^{\prime}$ of the relevant standard variety (such as in dialect speakers from the (former) German language enclaves in East Europe or in the former Soviet Union who remigrate into Germany. (Auer 2007, S. 111)

Zwei wichtige Charakteristika werden deutlich. Erstens handelt es sich um unterschiedliche Varietätenrepertoires: Die Zugewanderten aus deutschen Sprachinseln verfügen - im Unterschied zur autochthonen Bevölkerung in Deutschland - über Kompetenzen in nur einer deutschen Varietät - ihrem Basisdialekt. Zweitens entsteht eine neue soziolinguistische Konstellation für diese Basisdialekte: Die „dachlosen Außenmundarten“ (Sprachinseldialekte in der Definition von Kloss 1976) werden nach der Remigration durch die deutsche Gegenwartssprache ,überdacht“. Die beiden Charakteristika der Migrantengruppe betreffen ihre mitgebrachten Dialekte, d.h. die im Herkunftsland im informellen, familiären Alltagssprachgebrauch tatsächlich verwendeten Kommunikationsvarietäten. Was jedoch offen bleibt, sind der Status und die Funktion der deutschen Standardsprache bzw. des Hochdeutschen bei diesen Minderheiten. Für eine adäquate Beschreibung ihrer sprachlichen Migrationskonstellation ist diese Frage jedoch äußerst relevant, denn es handelt sich um den öffentlichen, von außen wahrnehmbaren Teil der Kommunikation, um „das Deutsch“ dieser Migrantengruppe. Die deutsche Standardsprache war bei der Einwanderung zwar keine aktiv verwendete Kommunikationsvarietät, ein wichtiger Faktor muss jedoch in Erwägung gezogen werden: Standarddeutsch (bzw. Hochdeutsch) war bei den deutschen Minderheiten in Osteuropa präsent, da es in der Regel als „Fremdsprache“ in der Schule erworben wurde. Allerdings handelte es sich bei dieser Form des Deutschen nicht um eine „reguläre“ Fremdsprache. Das Hochdeutsche wurde von den osteuropäischen Min- 
derheiten auf der Basis von deutschen Dialekten ${ }^{2}$ erworben und war in den deutschen Sprachinseln keine im Alltag aktiv verwendete Sprachvarietät. Die Sprachminderheiten entwickelten jedoch immer bestimmte Einstellungen gegenüber ihrer „hochdeutschen Muttersprache“. Aus diesem Grund ist bei den Einwanderern aus Osteuropa im Unterschied zu anderen Zuwanderungsgruppen in Deutschland eine differenzierte Attitüdenstruktur anzunehmen. ${ }^{3}$

Dieser Faktor spielte offensichtlich eine relevante Rolle bei der sprachlichen Integration und bei der Entwicklung des öffentlichen Standardsprachgebrauchs der Aussiedler. Bei der im IDS-Projekt „Sprachliche Integration von Aussiedlern“ (Berend 1998) durchgeführten soziolinguistischen Befragung von mehr als 100 Probanden der Einwanderungsgeneration hat sich gezeigt, dass es sich mehrheitlich um positive Einstellungen gegenüber der hochdeutschen Standardsprache handelte. Es stellte sich heraus, dass für den externen Sprachgebrauch die deutsche Standardsprache in der Einstellung der Aussiedlermigranten aus der Sowjetunion zu Beginn des Aufenthalts in Deutschland die einzige Alternative war. Sowohl die mitgebrachten russlanddeutschen Dialekte als auch die regional „gefärbten“ einheimischen Varietäten des Deutschen wurden als (mögliches) Kommunikationsmittel abgelehnt. ${ }^{4}$

Ausgehend von dieser Feststellung ist anzunehmen, dass unter Bedingungen des permanenten Sprachkontakts bestimmte Zusammenhänge zwischen den festgestellten positiven Attitüdenstrukturen, dem Varietätenwandel und der anzunehmenden längerfristigen Akkommodation im Zielland bestehen. Die ersten Ergebnisse des Projekts „Migrationslinguistik“ zeigen, dass im Sprachgebrauch und im Varietätenrepertoire der Einwanderungsgeneration in den zwei Jahrzehnten ihres Aufenthalts in Deutschland bestimmte Wandelprozesse stattgefunden haben, die auf den neuartigen Sprachkontakt zwischen Sprachinselvarietäten und deutschen autochthonen Varietäten und die sprachlich-soziolinguistische Situation in Deutschland zurückgehen.

In Bezug auf das Deutsch der Aussiedler der Einwanderungsgeneration stellen sich aktuell folgende Fragen:

Hier handelt es sich um verschiedene deutsche „Sprachinseldialekte“ des entsprechenden Herkunftslandes.

3 Leider wurde die Attitüdenstruktur im russischen Herkunftsland nicht erforscht. Ich beziehe mich hier auf eigene Erfahrungen aus deutschsprachigen Inseln in Russland. Zahlreiche Untersuchungen zu dieser Frage liegen dagegen für Ungarn vor. Vgl. dazu die bibliographischen Hinweise in Knipf-Komlósi (2011).

4 Das wurde auch durch andere Untersuchungen bestätigt. So wurden sogar Versuche unternommen, insbesondere von sehr erfolgsorientierten Müttern, den Gebrauch des Hochdeutschen rigoros in der ingroup-Kommunikation der Migrantenfamilie zu ,installieren“ (vgl. z.B. Diener 2004). 
(i) Ist Sprachwandel in real time festzustellen und wie ist er in die Sprachsituation des Einwanderungslandes einzuordnen?

(ii) Welche Hinweise geben die durchgeführten real time-Analysen auf das aktuelle Sprachrepertoire der Einwanderungsgeneration?

(iii) Bilden sich neue Regionalvarietäten des Deutschen aus?

Um Antworten auf diese Fragen zu bekommen, wurden mit einer Gruppe der ursprünglichen Probanden aus dem IDS-Aussiedlerprojekt (Berend 1998) seit 2009 Neuaufnahmen durchgeführt und mit den Daten der 1990er Jahre kontrastiert. Entsprechend der These des ingroup-outgroup-bedingten Sprachgebrauchs bei Migranten (Clyne/Eisikovits/Tollfree 2002) wurden Daten in drei unterschiedlichen Situationstypen erhoben:

\begin{tabular}{|l|l|l|l|}
\hline $\begin{array}{l}\text { Daten- } \\
\text { erbebung }\end{array}$ & \multicolumn{1}{|c|}{ ingroup } & \multicolumn{2}{|c|}{ outgroup } \\
\hline $\begin{array}{l}\text { Phase I } \\
\text { 1992-1994 }\end{array}$ & $\begin{array}{l}\text { internes Gespräch } \\
\text { mit Verwandten der } \\
\text { älteren Generation } \\
\text { der eigenen Migran- } \\
\text { tengruppe }\end{array}$ & $\begin{array}{l}\text { informelles Gespräch } \\
\text { mit einer autochthonen } \\
\text { Sprecherin der aufneh- } \\
\text { menden Region }\end{array}$ & $\begin{array}{l}\text { formelles Interview } \\
\text { mit einem Sprecher } \\
\text { der Standardsprache } \\
\text { in institutioneller Um- } \\
\text { gebung }\end{array}$ \\
\hline $\begin{array}{l}\text { Phase II } \\
\text { ab 2009 }\end{array}$ & $\begin{array}{l}\text { internes Gespräch } \\
\text { (wie oben) }\end{array}$ & $\begin{array}{l}\text { informelles Gespräch } \\
\text { (wie oben) }\end{array}$ & $\begin{array}{l}\text { formelles Interview } \\
\text { (wie oben) }\end{array}$ \\
\hline
\end{tabular}

Diese Erhebungsmethode erlaubt unterschiedliche Zugänge zum Untersuchungsgegenstand. Durch den Vergleich der Daten aus der ersten Aufnahmephase (1992) und der Daten aus dem Jahr 2009 (Längsschnittdaten) können Veränderungen in real time über den Zeitraum von knapp 20 Jahren festgestellt werden. Der Vergleich innerhalb jeder Aufnahmephase (Querschnittsdaten) ermöglicht die Erfassung der Differenzierungen innerhalb des Varietätenrepertoires der Sprecher zum Zeitpunkt der Aufnahmen. Im Folgenden beschäftige ich mich zunächst mit der Analyse der Längsschnittdaten zum jeweiligen Zeitpunkt: den Ausgangsvarietäten bei der Einwanderung und nach 18 Jahren Aufenthalt in Deutschland (Abschnitt 2). Im Abschnitt 3 geht es um das aktuelle Varietätenrepertoire der Einwanderungsgeneration. Es wird untersucht, ob gruppenexternes Sprachverhalten der Migranten situationsabhängige Unterschiede aufweist (Analyse der Querschnittsdaten). Abschließend werde ich die Frage diskutieren, wie der durch den Sprachkontakt hervorgerufene Varietätenwandel zu bewerten ist und ob er zu einer Art neuer Regionalsprache des Deutschen führt (Abschnitt 4). 


\section{Varietätenwandel in real time - Analyse der Längsschnittdaten}

Im Folgenden konzentrieren sich die Ausführungen zunächst auf die Analyse der Längsschnittdaten einer Dialektsprecherin, die 1992 aus einer deutschen Sprachinsel im Altai-Gebiet (Sibirien) im Alter von 31 Jahren nach Deutschland eingewandert ist. Es handelt sich um die Sprecherin S. In der Herkunftsregion der Probandin waren Dialekte rheinfränkischsüdfränkischer Provenienz verbreitet; seit der Migration ist die Probandin in der rheinfränkisch-moselfränkischen Dialektregion im mittleren Saarland ansässig.

Im Folgenden werden vier Fallbeispiele dieser Sprecherin analysiert. Zuerst geht es um die Ausgangsvarietäten der Sprecherin (Fallbeispiel 1 und 2 aus der ersten Aufnahmephase im Jahr 1992), danach um die aktuellen Varietäten (Fallbeispiel 3 und 4 aus dem Jahr 2009).

\subsection{Ausgangsvarietät: „Intendiertes Hochdeutsch“}

Das erste Fallbeispiel „Sprachkurs“5 ist ein Auszug aus dem Transkript des Interviews, das von einem Sprecher der Standardsprache mit der Probandin kurz nach ihrer Einreise in einem institutionellen Kontext durchgeführt wurde. Thematischer Schwerpunkt des Interviews ist die Herkunft der Familie der Sprecherin und ihre ersten Eindrücke in Deutschland. Im vorliegenden Auszug geht es um den Sprachkurs für Aussiedler, der von der Sprecherin S. und ihrem Mann zum Zeitpunkt des Interviews besucht wird.

(1) Sprachkurs

01 I: haben sie in deutschland einen sprachkurs dann besucht oder $\uparrow j a \uparrow$

Sprachbeispiel „Sprachkurs“ - Interview in institutioneller Umgebung (1992, kurz nach der Einreise): russlanddeutsche Sprecherin S. (31 Jahre); I = Interviewer B.U., Sprachwissenschaftler. Zur Transkription siehe Anhang. 
In diesem Beispiel fällt zunächst auf, dass es sich zweifellos um eine im Unterricht Deutsch als Fremdsprache erlernte deutsche Varietät handelt, also um eine ,russische DaF-Varietät“, die an sowjetischen allgemeinbildenden Schulen erworben werden konnte. ${ }^{6}$ Dafür sprechen die sehr typischen phonetischen Merkmale wie die volle Aussprache von Nebentonvokalen bei Substantivendungen und im Präfix ge- der Partizipien, die nichtvokalisierte Aussprache des auslautenden /r/ u.a.

Andererseits gibt es Formen, die bei russischen native speakers beim Erlernen des Deutschen nicht vorkommen. Einige davon sind z.B.: $e$ - und n-Apokope (bätt ,hätte“; kenne „,können“); $a$-Verdumpfung ( $j o$, ,ja"); 0 -Entrundung (kenne „können“); das Dialektlexem no „,nachher, dann“; binnendeutsche Konsonantenschwächung (konnden „konnten“) bzw. umgekehrt die stimmlose Aussprache von Plosiven. Im grammatischen Bereich ist das das auffällige, typisch dialektale Beispiel der Kasusmischung (mit mich , mit mir ${ }^{\circ}$. Diese Dialektformen deuten darauf hin, dass es sich nicht um einen native speaker des Russischen und nicht nur um eine DaF-Sprecherin handelt, sondern auch um eine Sprecherin mit deutschen Dialektkenntnissen.

Auffällig ist, dass die Sprecherin sich konsequent bemüht, Hochdeutsch zu sprechen. Diese Varietät ist demnach eine Form des intendierten Hochdeutsch, wie es auch anderswo bei dialektkompetenten Sprechern im deutschen Sprachraum nicht unüblich ist. ${ }^{8}$ Allgemein gelingt es der Sprecherin relativ gut, im Laufe des gesamten ca. einstündigen Interviews, eine ,hyperhochdeutsche" Varietät zu realisieren.

\subsection{Ausgangsvarietät: outgroup-Gespräch}

Zu ähnlichen Ergebnissen kommen wir bei der Analyse des zweiten Fallbeispiels: „Zu nix gekommen. “9 Bei diesem Beispiel handelt es sich um Daten ganz anderen Typs: Es ist ein Gespräch zwischen derselben Probandin S. und der einheimischen Gesprächspartnerin K.

Hier greife ich auf meine eigenen Erfahrungen aus der Praxis des DaF-Unterrichts in der Sowjetunion zurück. Weitere Beispiele für diese Varietät finden sich in Berend (2011).

7 Es gibt in den Texten auch Wortstellungsbesonderheiten, die sowohl als Merkmale des nonnative-Hintergrunds aber auch als Hinweise auf den dialektalen Hintergrund betrachtet werden können (wie z.B. mein mann dä bätt jo kenne machen spracbkurs). Syntaktische Merkmale des Russischen und des gesprochenen Deutsch (Dialektsyntax) fallen in bestimmten Bereichen der Wortstellung weitgehend zusammen. Jedig (1970) untersuchte die möglichen Auswirkungen des Russischen auf die Syntax des Niederdeutschen in Russland (vgl. Jedig 1970, II, S. 163-172 und Berend/Jedig 1991, S. 182-183).

8 Siehe z.B. besonders Häcki Buhofer/Burger (1998) für sehr ähnliche Erscheinungen in der Schweiz.

$9 \quad$ Sprachbeispiel „Zu nix gekommen“ - nachbarschaftliches Gespräch (aufgenommen in H. 1992): S. = russlanddeutsche Sprecherin (30 Jahre); K. = einheimische Nachbarin (55 Jahre). 
Die Probandin S. und Frau K. sind seit sechs Monaten Nachbarn in einem Reihenhaus. In einem Vorgespräch konnte ich feststellen, dass die Familie K. sehr zufrieden ist mit den neuen „,russischen“ Nachbarn. Sie kämen gut miteinander aus und unterhielten gute nachbarschaftliche Beziehungen. Die interessierte und hilfsbereite Saarländerin nimmt aktiv teil an allen Angelegenheiten der neuen Nachbarn, tritt oft als Vermittlerin und Verhaltensratgeberin in allen neuen komplizierten Lebensfragen auf (z.B. welches Kleid man zur Kommunion trägt usw.).

In dem aufgezeichneten Gespräch, das zu Hause bei Frau K. stattfindet, ist vor allem ein Thema auf der Tagesordnung: Unsere Probandin beantwortet Fragen und erklärt ihr Leben in Russland. Im Gespräch geht es darum, dass es in ihrem Heimatdorf in der Sowjetunion vor der Auswanderung doch noch erträglich war, da sie ihr eigenes Vieh hielten und mehr oder weniger alles selbst hergestellt hätten, anders als in den Städten, wo es so gut wie nichts an Lebensmitteln in den Geschäften zu kaufen gab. Nahezu das gesamte Gespräch besteht aus Vergleichen, wie es in Russland war bzw. in Deutschland ist.

Im Kontext des folgenden Ausschnitts fragt Frau K. danach, ob Frauen in Russland alle gearbeitet hätten. Darauf antwortet die Probandin, dass in ihrem Dorf alle Frauen gearbeitet hätten. Frau K. gibt daraufhin den Kommentar ab, dass der Staat trotzdem zu „nix gekommen“ sei.

(2) Zu nix gekommen

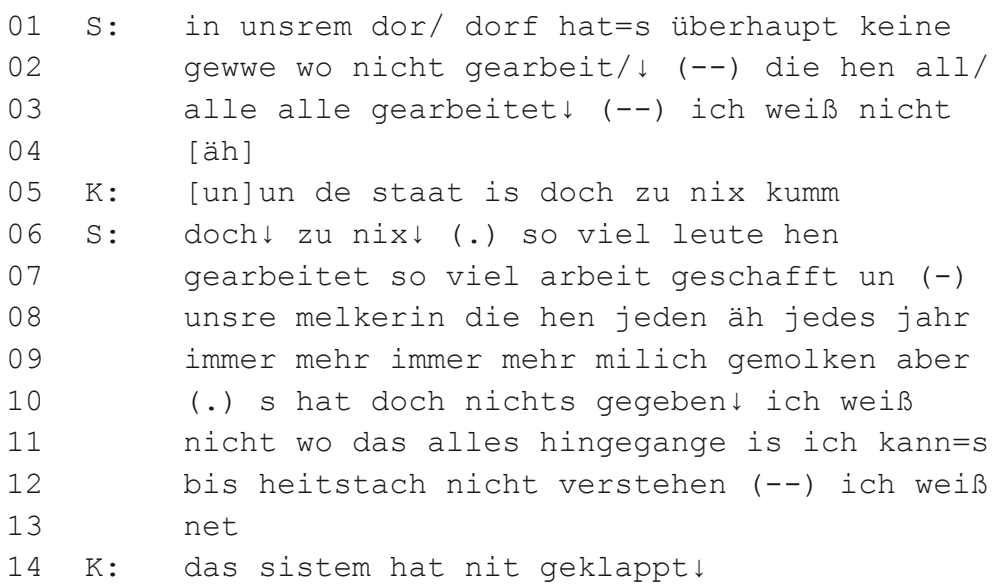

Obwohl die Fragen und Beiträge von Frau K. neben Hochdeutsch auch im Regionaldeutsch (Saarländisch) erfolgen, gebraucht die Probandin S. wiederum das intendierte Hochdeutsch wie auch im Beispiel (1). Alle für das Beispiel (1) genannten Merkmale der DaF-Variante sind auch in diesem Beispiel vorhanden. 
Nur vereinzelte Dialektwörter wie z.B. heitstach (heutzutage) oder net (nicht) und einzelne nicht hochdeutsche Aussprachevarianten wie milich (Milch), unsre (unsere) oder gewwe (geben) erinnern an die dialektale Varietät. In Zeile 6 passt sich die Probandin an die Gesprächspartnerin K. an, wenn sie die gesprochensprachliche Form nix verwendet, gebraucht dann aber nur ein paar Zeilen weiter erneut die standardsprachliche Variante nichts.

Dafür, dass es sich um eine intendiert hochdeutsche Varietät handelt, sprechen auch die Mischlexeme bzw. Zwischenlexeme, d.h. nur zum Teil veränderte, standardsprachlich intendierte Formen. Das Lexem gegewen im folgenden Beispiel (an einer anderen Stelle in der Aufnahme) ist ein solcher Fall, bei dem die Sprecherin eine Mischform aus gewne und gegeben gebraucht (Z. 4).

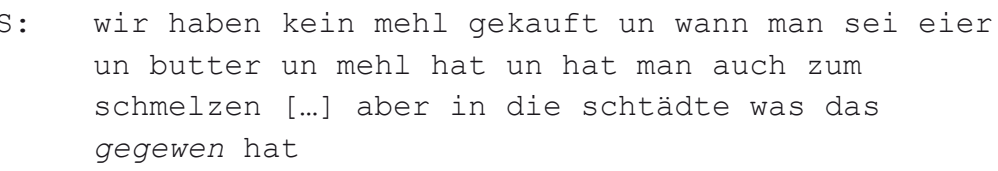

Weitere Beispiele für häufig auftretende Muster des intendierten Hochdeutsch im outgroup-Gespräch sind bei der Probandin S. Mischformen wie frachen „fragen“ (dial. frooche), gehert „gehört" (dial. gheert), gwesen „gewesen“ (dial. gwest), sachen ,sagen“ (dial. saache), scbtehnen „stöhnen“ (dial. schteene). In diesen und ähnlichen Beispielen, die hoch frequent sind, kookkurrieren standardsprachliche und dialektale Merkmale. ${ }^{10}$

Für die später zu untersuchende Frage des Wandels im Varietätenrepertoire ist wichtig festzuhalten, dass die Sprecherin sich unabhängig vom Gesprächspartner konsequent an das intendierte Hochdeutsch hält. Sie macht keinen Unterschied zwischen dem unbekanntem Interviewer, der konsequent Hochdeutsch spricht, und der ihr bereits einigermaßen gut bekannten Nachbarin, die in diesem Gespräch zwar auch überwiegend eine hochdeutsch-angenäherte Varietät verwendet, aber auch häufig in den Regionalstandard bzw. in die saarländische Regionalvarietät wechselt.

\subsection{Ausgangsvarietät outgroup-Gespräch nach 18 Jahren}

Das Transkript „Silberne Hochzeit" ${ }^{\text {"11 }}$ (Sprachbeispiel 3) ist ein Auszug aus einem einstündigen Gespräch zwischen den beiden eben analysierten Sprecherinnen nach 18 Jahren - der Probandin S. und der Nachbarin Frau K. -, das - ähnlich wie im vorhergehenden Beispiel - wieder in der Wohnung von Frau K. stattfindet.

10 Mischformen dieses Typs sind ein wichtiges Merkmal der intendierten Hochdeutsch-Varietät aller bisher untersuchten Sprecher dieser dialektgeprägten Migrantegruppe.

11 Fallbeispiel „Silberne Hochzeit“ - informelles Gespräch 2009: S. = russlanddeutsche Sprecherin; K. = befreundete einheimische Bekannte. 
Die beiden Gesprächsteilnehmerinnen wohnen seit einigen Jahren zwar nicht mehr in Nachbarschaft, da die russlanddeutsche Familie nach dem Kauf eines Hauses in einen anderen Ort umgezogen ist, sie unterhalten aber immer noch gute freundschaftliche Beziehungen. Im aktuellen Auszug handelt es sich um eine Erzählung der Probandin S. darüber, dass sie und ihr Mann in diesem Jahr Silberne Hochzeit haben, aber sich nicht leisten können, sie zu feiern.

\section{(3) Silberne Hochzeit}

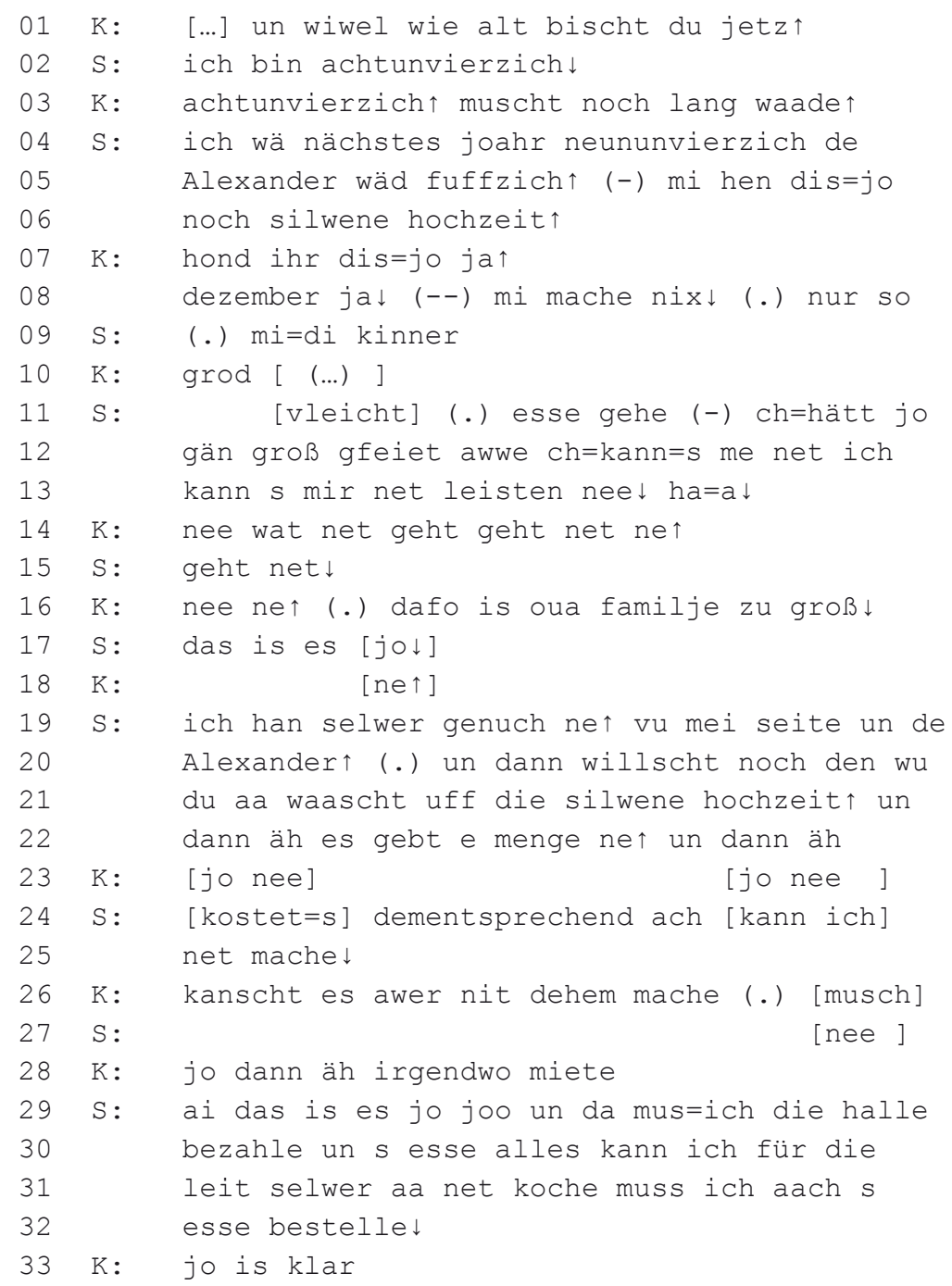




$\begin{array}{lll}34 & \mathrm{~S}: & \text { nu un } \mathrm{s}=\text { kostet dann ne nee ha=a ich han } \\ 35 & & \text { gsacht mi kenne aach mit unsre kinne is net } \\ 36 & & \text { schlechter äh (.) gehe=me ese oder so oder } \\ 37 & & \text { oder [dehem is ja ach egal] } \\ 38 & \mathrm{~K}: & \text { [owe dehem jor mache ouch scheena } \\ 39 & \mathrm{~S}: & \text { mache joo } \\ 40 & \mathrm{~K}: & \text { ne } \\ 41 & \mathrm{~S}: & \text { ansonsten } \uparrow \\ 42 & \mathrm{~K}: & \text { klar }\end{array}$

Zunächst muss festgehalten werden, dass das Beispiel durch seinen stark ausgeprägten dialektalen Eindruck auffällt. Betrachten wir den Auszug vom Gesichtspunkt der vorkommenden Dialektmerkmale etwas genauer, wird klar, dass es sich beim Großteil der Merkmale um typische Eigenschaften des russlanddeutschen Herkunftsdialekts handelt. Einige Beispiele sollen zur Veranschaulichung der Dialektaussprache genügen: $a$-Verdumpfung (Johr); b-Spirantisierung (silwene); nd-Assimilation (kinner); $n$-Apokope (mache, esse, gehe); e-Apokope (bätt); e-Synkope (gfeiert); s-Palatalisierung (willscht, warscht); au-Monophthongierung (aach); eu-Entrundung (leit). Außerdem kommen häufig lexikalische Dialektvarianten vor: mir (wir), hen (haben), net (nicht).

Der Herkunftsdialekt der Probandin mit pfälzisch-südfränkischer Provenienz weist auf der strukturellen Ebene viele Gemeinsamkeiten mit der lokalen autochthonen Mundart des rheinfränkischen Teils des Saarlands auf. Dass es sich bei den Dialektmerkmalen jedoch nicht nur um solche aus dem Herkunftsdialekt handelt, erfahren wir ab Zeile 19: Hier werden die neuen Varianten gebraucht, die die beiden westmitteldeutsch basierten Dialekte unterscheiden und die als saarländische Marker betrachtet werden können. ${ }^{12}$ Dies sind das Hilfsverb ban ,(ich) habe“ sowie die Diskurspartikeln ne (Z. 19) und ai (Z. 29). Auf den ersten Blick erscheint der Gebrauch dieser Varianten des Saarländischen nicht überraschend: Er kann im Rahmen der accommodation theory als short term accommodation innerhalb eines Interaktionsakts gedeutet werden (nach Auer/Hinskens 2005 die erste Stufe der Akkommodation die Anpassung an den unmittelbaren Gesprächspartner). Tatsächlich verwendet die Gesprächspartnerin K. in der Regel das wespfälzische, in der saarländischen Region des Rheinfränkischen übliche Hilfsverb han, hier in der 2.P.Pl. in Zeile 7: hond ihr dies=jo ja? „habt ihr dieses Jahr, ja?"“. Auch die beiden Diskurspartikeln ne und $a i$ werden von der saarländischen Ge-

$12 \quad$ Zu saarländischen Varianten siehe Bellmann/Herrgen/Schmidt (2002) und Ramge (1982). Vgl. auch Barden/Großkopf (1998) zur sprachlichen Akkommodation der Umsiedler aus den neuen Bundesländern im Saarland. 
sprächspartnerin häufig verwendet. ${ }^{13}$ An dieser Stelle könnte angenommen werden, dass unsere Probandin sich dieser aktuellen Variantenverwendung der saarländischen Sprecherin anpasst und dass es sich um short term accommodation handelt (Auer 2000). ${ }^{14}$ Dass dies jedoch nicht der Fall ist, wird sich bei der folgenden Analyse des Varietätenrepertoires der Sprecherin im Abschnitt 3 zeigen.

Dieses Ergebnis steht im Einklang mit den Ausführungen in Auer/ Hinskens (2005) bezüglich der Rolle der interpersonal accommodation. Danach orientieren sich Sprecher häufig nicht an den konkreten vorangegangenen sprachlichen Äußerungen ihrer Gesprächspartner, sondern eher an den eigenen stereotypen Vorstellungen, die sie über bestimmte Gesprächskonstellationen entwickeln. Welche Bedeutung das für das Varietätenrepertoire der Migranten hat, wird im Folgenden noch ausführlich diskutiert.

Auffällig für einheimische Deutschsprecher könnten die ungewöhnlichen Kookkurrenzmuster sein, die im Transkript belegt sind. Das sind erstens Kookurrenzen auf der Satzebene. Zu dem Typ gehört z.B. der Ausdruck kann s mir net leisten (Z. 13). Hier würde man statt des standardsprachlichen leisten eher die Dialektvariante leischte bzw. leiste (mit n-Apokope) erwarten. Es handelt sich hier aber um ein neues Wort, das im russlanddeutschen Herkunftsdialekt nicht existiert, und aus diesem Grund wird wohl die standardsprachliche Lautung produziert.

Innerhalb der Präpositionalphrase für die leit (Z. 30f.) wäre nach den standardsprachlichen Elementen für das Bezugsnomen eher die Standardaussprache angebracht, die Sprecherin gebraucht jedoch die Dialektvariante, möglicherweise weil es sich hier um ein sehr häufig verwendetes typisches Dialektlexem mit $e n$-Entrundung und $e / n$-Apokope (,Leute) handelt. Auch innerhalb der Präpositionalphrase vu mei seite (Z. 19) ist das heterogene Kookkurrenzmuster eher ungewöhnlich: Nach dialektaler Präposition und Pronomen passt bei dem Bezugsnomen Seite das nicht apokopierte - $e$ nicht in das Bild der üblichen (homogenen) regionalsprachlichen Kookkurrenzmodelle.

Zweitens sind mehrere Kookkurenzauffälligkeiten auf der Wortebene belegt. Dass die Sprecherin im intendierten Hochdeutsch in der ersten Aufnahmephase viele Mischformen auf der Wortebene verwendete, wurde oben bereits dargestellt. Die Analyse der Gesamtaufnahme für 2009 ergab ähnliche Kookkurrenzmuster, häufig für neue (im Herkunftsdialekt fehlende) Lexeme wie z.B. die heterogene Kombination von gerundetem $\ddot{u}$ und der b-Spirantisierung in Üwwerdacbung.

Ich verzichte an dieser Stelle auf die genaue Einordnung der Diskurspartikel ne dahingehend, $\mathrm{ob}$ es sich um eine regionale Dialektvariante oder eine Form des gesprochenen Standards bzw. Gebrauchsstandards handelt, vgl. dazu Berend (2005) und Kleiner et al. (2011). Vgl. jetzt auch Deppermann/Kleiner/Knöbl (i.Dr.).

Diese Partikel scheint allerdings auch ein typisches Merkmal in der saarländischen Region zu sein.

14 Vgl. auch die Synchronisierungstheorie von Schmidt/Herrgen (2011). 
Gerade für solche „neuen“ Lexeme scheinen (noch) keine Kookkurrenzrestriktionen zu existieren, da gleiche Lexeme in unterschiedlichen Kombinationen von Merkmalen vorkommen. So erscheint das Lexem überwiesen in zwei „Kookkurrenzversionen“, wobei beide für den entsprechenden einheimischen/autochthonen Regionalgebrauch aufgrund von Kookkurrenzrestriktionen eher unüblich sind: Die erste Version stellt eine Kombination von gerundetem $\ddot{u}$, der $b$-Spirantisierung und $n$-Apokope dar: üwwerwiese; bei der zweiten verbinden sich die $\ddot{u}$-Entrundung und das Ausbleiben der $n$ Apokope: inwerwiesen. (Auf die Frage der Kookkurrenzen und ihrer Rolle bei der Bewertung der Migrantenvarietäten komme ich im Abschnitt 4 zurück.)

\subsection{Ausgangsvarietät ,intendiertes Hochdeutsch“ nach 18 Jahren}

Beim letzten zu analysierenden Fallbeispiel (4) „Leintuch“"15 handelt es sich um einen Auszug aus dem auf Hochdeutsch geführten Interview mit der Probandin S. im Jahr 2009. ${ }^{16}$ Es geht wieder um Fragen bezüglich der sprachlichen und gesellschaftlichen Integration der Familie in Deutschland wie im Beispiel (1) zu Beginn des Abschnitts. Im vorliegenden Auszug erzählt die Probandin von einem Fall, der die Ähnlichkeiten zwischen ihrem eigenen Herkunftsdialekt und der Sprechweise im Saarland veranschaulichen soll.

(4) Leintuch

01 I: ja das wollte ich hören dass gewisse

02 dialekteigenheiten im saarland und bei ihnen

03 in russland ähnlich sind [...

$04 \mathrm{~S}$ : in unserem dorf ne $\uparrow$ wo mir ware ja ja

15 Fallbeispiel „Leintuch“ - Interview (2009): S. = russlanddeutsche Sprecherin; I. = standarddeutsch sprechender Interviewer.

16 Der Interviewer ist der Probandin nur aus einem früheren Interview bekannt. 
Anders als in 1992 ist dieses Beispiel varietätenmäßig uneinheitlich und auf den ersten Blick widerspruchsvoll. Auffällig ist zunächst der Gebrauch von Dialektvarianten. (Es handelt sich ja um ein „Standardinterview“.) Die meisten Dialektphänomene stammen aus dem Herkunftsdialekt. Es seien hier einige genannt: verbreitete Dialektlexeme (mir „wir“, net „nicht“, hen „haben“), $a$-Verdumpfung ( jo ,ja“, do „da“, mol „mal"), Lenisierung (konnde „konnten“, leinduch „Leintuch“), b-Spirantisierung (awwe „aber“), g-Spirantisierung (sache „sagen"), s-Palatalisierung (hascht „hast"), au-Monophthongierung (aach ,auch“), e-Synkope (gsagt ,gesagt"), n-Apokope (komme ,gekommen'). ${ }^{17}$ Beim ,intendierten Hochdeutsch“" in 1992 wurden diese Dialektphänomene „unterlassen“. Auch hier gäbe es die Möglichkeit der Unterlassung; z.B. in Bezug auf die n-Apokope gäbe es dafür schon in diesem kurzen Abschnitt zehn mögliche Fälle, doch die Sprecherin gebraucht diese Lexeme ohne die Endung -n: musste, erlediche, konnde, verstanne, komme, wusste, wisse und sache.

Neben den Merkmalen aus dem Herkunftsdialekt ${ }^{18}$ werden auch neue Varianten des lokalen Dialekts der Aufnahmeregion (des Saarländischen) verwendet. Im Text erscheinen folgende auffällige saarländische Marker: gesat: ohne $e$-Synkope mit Schwund des $g$ vor $t$, han: typische Form für ,haben“, kriet: ohne Präfix ge- und mit Schwund des $g$ vor $t$, ne: Diskurspartikel. ${ }^{19}$

Trotz der Verwendung von dialektalen Merkmalen aus dem Herkunftsdialekt und dem lokalen saarländischen Dialekt handelt es sich bei diesem Beispiel um eine vom Hochdeutschen beeinflusste bzw. am Hochdeutschen ausgerichtete Varietät. Davon zeugen zahlreiche Merkmale der Standardsprache.

17 Bei einigen Merkmalen könnte es sich auch um autochthone Varianten der saarländischen Region handeln. Die Daten aus der ingroup-Kommunikation (interne Kommunikation kurz nach der Einwanderung) dienen hier als Zuordnungs- und Unterscheidungsgrundlage.

18 Für den Leser auffällig können natürlich auch andere Merkmale sein wie z.B. die auf den ersten Blick typologisch fremden Wortstellungen mit SVO entgegen der deutschen Verbklammer: mir ban jo ach am anfang kriet äh geschirr. Vgl. dazu aber Anmerkung 7.

19 Die Diskurspartikel ne (nicht? nicht wahr?) wird von der Probandin in diesem kurzen Auszug fünfmal verwendet (in den Zeilen 4, 10, 11, 15, 18). Das saarländische Hilfsverb han erscheint zweimal (in Zeile 8 und 13), obwohl der Hochdeutsch sprechende Interviewer diese Partikel und das Verb han nicht gebraucht. 
$\mathrm{Zu}$ den Standardlexemen, die erst in Deutschland neu erworben wurden und die im Dialekt nicht existierten, gehören im Text: erlediche, gemeinde, anträge, irgendwie, fall, hier. Dazu gehören auch typische Phraseolexeme, wie ab und $z u^{20}$

Auf der phonetischen Ebene macht sich die Hochdeutsch-Ausrichtung durch die „Unterlassung“"von möglichen typischen Dialektmerkmalen (wie am Anfang des Abschnitts beschrieben) bemerkbar. Das sind die nicht durchgeführten Entrundungen (deutsch, im Dialekt daitsch), Monophthongierungen (heißt, weiß, im Dialekt heescht, wees), Apokopen (Bettwäsche, im Dialekt Bettwäsch), Synkopen (Geschirr, im Dialekt gschärr) usw.

Der Text enthält außerdem auch eine im Material rekurrente Erscheinung, nämlich eine Formkorrektur in Richtung gesprochenes Standarddeutsch, und zwar beim unbestimmten Artikel: $n$ leinduch (,ein Leintuch“, Z. 19) für das dialektale $e=$ lainduch (Z. 18).

Kommen wir jetzt zu den Eigenschaften, die neu sind im Vergleich zur Phase 1. In Zeile 16 wird die Variable da in ihrer standardsprachlichen Variante da und in der Dialektform do verwendet: un da hen mir gsacht do feblt noch, d.h. bei der Fokussierung eines Zitats wird die Standardvariante verwendet, im Zitat wird die dialekttypische $a$-Verdumpfung durchgeführt. ${ }^{21}$ Hier handelt es sich um die intrasituative Variation zwischen der Standardsprache und dem Dialekt. Die Verwendung von dialektalen neben standardsprachlichen Formen führt zur typischen Standard/Dialekt-Variation, d.h. zur vertikalen Variation (Auer 2000).

Das Hilfsverb hen aus dem Herkunftsdialekt variiert mit der entsprechenden lokalen Variante han in der Äußerung: wie me hen bettwäsche kriet mir ban jo ach am anfang kriet geschirr (Z. 14). Die Verwendung von herkunftsdialektalen und neuen lokalen Varianten des Saarländischen stellt den zweiten Typ der intrasituativen Variation dar: die Dialekt/Dialekt-Variation bzw. die zwischendialektale oder horizontale Variation. ${ }^{22}$

Der dritte Typ der intrasituativen Variation kann in diesem Text gut am Beispiel der Partizipform gesagt veranschaulicht werden. Das Lexem gesagt kommt insgesamt in fünf verschiedenen Ausspracheversionen vor, wird in der standardsprachlichen Form jedoch nur vom Interviewer verwendet (Z. 20). Die Sprecherin gebraucht vier unterschiedliche Varianten: russlanddeutsch (gsagt, Z. 23), saarländisch (gesat, Z. 12) sowie zwei Zwischenfor-

20 Im Herkunftsdialekt werden dafür Umschreibungen bzw. auch russische Entsprechungen genutzt.

21 Beide Variationsbeispiele können auch als Belege für die diskurs-strukturierende Funktion von Variation gesehen werden (siehe Kallmeyer/Keim 1994).

$22 \mathrm{Zu}$ aktuellen Prozessen der vertikalen und horizontalen Konvergenz im deutschsprachigen Raum vgl. Auer (2000). Hier ist allerdings anzumerken, dass im Migrationsmodus die Kategorie „horizontale“ Variation sich verschieben kann, da für Migranten auch Regionalvarietäten der Einheimischen „Prestigevarietäten“ darstellen (können). Hierzu fehlen noch entsprechende Untersuchungen. 
men (gsacht und gsat, Z. 16, 21). Diese Mischformen zeigen, dass auch für dieses Beispiel die oben angestellten Überlegungen zu Kookkurrenz-Auffälligkeiten gelten.

Der Beispieltext vermittelt zwar auf den ersten Blick durch das Vorhandensein zahlreicher Dialektismen einen starken dialektalen Eindruck, doch handelt es sich auch hier, wie im vorhergehenden outgroup-Gespräch, um eine eher am Hochdeutschen ausgerichtete Sprachvarietät. Die Sprecherin ist sich der outgroup-Situation bewusst und verwendet eine nach ihren Vorstellungen angemessene ,hochdeutsche“ Varietät.

\subsection{Diachrone Frequenzanalysen}

Nach der Analyse der Fallbeispiele der Sprecherin S. werden im Folgenden einige Ergebnisse der Frequenzanalyse ausgewählter Variablen vorgestellt werden. Bei der Frequenzanalyse geht es darum, zu vergleichen, wie hoch der Dialektformanteil bestimmter Variablen in den untersuchten Hochdeutschvarietäten 1992 und 2009 ist. $^{23}$ Es handelt sich im Folgenden nur um Merkmale, für die der höchste prozentuale Anstieg der Dialektformen festgestellt wurde: die $s$-Palatalisierung, ei-Monophthongierung, $n$-Apokope, die Dialektlexeme net (nicht) und mir (wir). In Tabelle 1 sind die Ergebnisse der Auszählung in Bezug auf diese Merkmale dargestellt; in den markierten Spalten ist die Differenz aus der Längsschnittperspektive angegeben. Schon die Ausgangsvarietät im Interview 1992 war von verschiedenen Dialektmerkmalen durchsetzt, obwohl es sich um ,intendiertes Hochdeutsch“ handelt (vgl. Abschnitt 2.1).

\begin{tabular}{|c|c|c|c|c|c|c|c|}
\hline \multirow{4}{*}{ Variable } & \multicolumn{7}{|c|}{ Interview } \\
\hline & \multicolumn{3}{|c|}{1992} & \multicolumn{3}{|c|}{2009} & \multirow{3}{*}{$\begin{array}{c}\text { Diff. } \\
(\%)\end{array}$} \\
\hline & \multirow{2}{*}{$\begin{array}{c}\sum \\
\text { abs. }\end{array}$} & \multicolumn{2}{|c|}{ Dialektanteil } & \multirow{2}{*}{$\begin{array}{c}\sum \\
\text { abs. }\end{array}$} & \multicolumn{2}{|c|}{ Dialektanteil } & \\
\hline & & abs. & $\%$ & & abs. & $\%$ & \\
\hline $\begin{array}{l}\text { /st/-/št/ } \\
\text { (Palatalisierung) }\end{array}$ & 50 & 6 & 12,0 & 111 & 74 & 66,7 & $+54,7$ \\
\hline $\begin{array}{l}\mathrm{b} /-/ \mathrm{w} / \\
\text { (Spirantisierung) }\end{array}$ & 73 & 20 & 27,4 & 63 & 52 & 82,5 & $+55,1$ \\
\hline $\begin{array}{l}\text { /ei-e/ } \\
\text { (Monophthongierung) }\end{array}$ & 64 & 14 & 21,9 & 133 & 118 & 88,7 & $+66,8$ \\
\hline /-n/-/ø/n-Apokope & 218 & 93 & 42,7 & 469 & 375 & 80,0 & $+37,3$ \\
\hline nicht - net & 115 & 15 & 13,0 & 143 & 141 & 98,6 & $+85,6$ \\
\hline wir $-m i r$ & 74 & 23 & 31,1 & 152 & 146 & 96,1 & $+65,0$ \\
\hline
\end{tabular}

Tab. 1: Übersicht über den Anstieg des Dialektanteils bei 6 Variablen zwischen 1992 und 2009 in den Sprachdaten der Sprecherin S. im Situationstyp „Interview“

23 Eine Publikation der Gesamtergebnisse ist in Vorbereitung (vgl. Berend i.Vorb.). 
Aus Tabelle 1 geht hervor, dass 2009 der Dialektformanteil bei diesen Variablen noch massiv gestiegen ist. Die rechte Spalte zeigt die Differenz zwischen beiden Aufnahmephasen. Der Gebrauch der Variante net steigt z.B. um 85,6 Prozent und der der Variante mir um 65 Prozent (vgl. Tab. 1). Damit ist klar, dass im Fall dieser Variablen die Sprecherin 2009 fast durchgehend dialektale Varianten der Aufnahmeregion gebraucht.

Ähnliche Ergebnisse liegen auch für das outgroup-Gespräch vor. Abbildung 1 zeigt die Veränderungen (Mittelwert) in Bezug auf den Dialektanteil im Gesamtkorpus 1992 und 2009 in den drei Erhebungssituationen. Der Dialektanteil im outgroup-Gespräch steigt mit 1,3 Prozent (Mittelwert) zwar nicht so deutlich wie im Interview, aber auch im Gespräch zwischen der Probandin und der einheimischen Sprecherin im Jahr 2009 erhöht er sich leicht (von 72,6 auf 73,9 Prozent), während im Interview der Anstieg 18,4 Prozent beträgt: von 51,4 auf 69,8 Prozent.

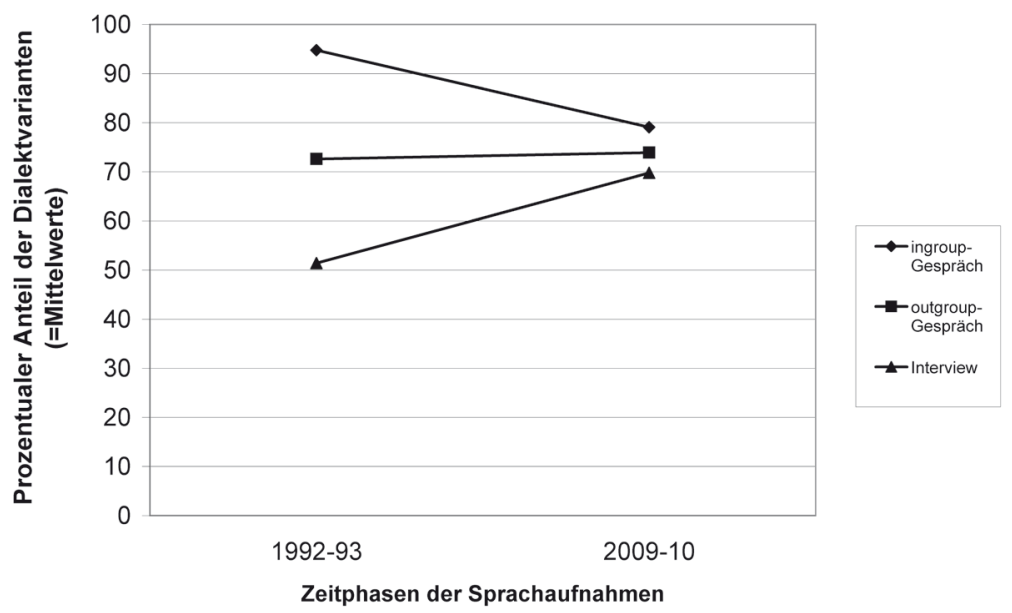

Abb. 1: Entwicklung des Dialektanteils in den Sprachdaten der Sprecherin S. in den zwei Situationstypen outgroup-Gespräch und Interview

\subsection{Sprechervergleich}

Bisher wurde nur der Sprachgebrauch der Sprecherin S. analysiert. Um festzustellen, ob es sich nicht (nur) um individuelle Sprachentwicklungen handelt, werden im Folgenden die Ergebnisse der Frequenzanalyse in Bezug auf zwei weitere vollständig ausgewertete Sprecherinnen mit ähnlichen soziolinguistischen Ausgangsdaten und vergleichbaren Aufnahme-/Gesprächssituationen analysiert (vgl. Tab. 2). 


\begin{tabular}{|l|r|r|r|r|r|r|}
\hline Sprecher & \multicolumn{2}{|c|}{$\begin{array}{c}\text { outgroup- } \\
\text { Gespräch }\end{array}$} & Diff. & \multicolumn{2}{|c|}{ Interview } & Diff. \\
\hline & 1992 & $2009 / 10$ & & 1992 & $2009 / 10$ & \\
\hline S 1 & 72,6 & 73,9 & $+1,3$ & 51,4 & 69,8 & $+18,4$ \\
\hline S 2 & 57,9 & 66,5 & $+8,6$ & 43,9 & 46,6 & $+2,7$ \\
\hline S 3 & 64,8 & 55,7 & $-9,1$ & 38,5 & 46,7 & $+8,2$ \\
\hline
\end{tabular}

Tab. 2: Die Entwicklung des Dialektanteils bei drei Sprecherinnen in zwei untersuchten Situationen (Mittelwerte in Prozent)

Bei den beiden ersten Sprecherinnen zeigt sich die Zunahme des Dialektanteils in beiden Situationen, d.h. es liegen keine situationstypischen Unterschiede vor. Bei der dritten Sprecherin zeigt sich eine Reduktion der Dialektvarianten im outgroup-Gespräch und eine Zunahme im Interview. ${ }^{24}$ Was jedoch auffällig ist: In Bezug auf unterschiedliche Variablen weisen die untersuchten Sprecher unterschiedliche Tendenzen auf, das muss hier besonders hervorgehoben werden. Beim Sprechervergleich konnten zwei wichtige Ergebnisse festgemacht werden:

1) Der diachrone Abbau verlief selektiv: Einzelne Variablen wurden von einigen Sprechern beibehalten, die anderen wurden abgebaut, und zwar zu jeweils unterschiedlichem Grad.

2) Alle untersuchten Sprecher tendieren dazu, keine Situationsdifferenzierungen vorzunehmen: Wenn eine Variable abgebaut wird, dann grundsätzlich durchgehend in allen analysierten externen Gesprächssituationen und z.T. - überraschenderweise - auch in der ingroup-Kommunikation (d.h. in Gesprächen mit Angehörigen der eigenen Migrantengruppe). ${ }^{25}$

Als Beispiel für die Punkte 1 und 2 sei hier die Variable wann/wenn für vier Sprecherinnen in drei Situationstypen (ingroup-Gespräch, outgroup-Gespräch und Interview) angeführt. So wurde die Dialektvariante wann (für, wenn ${ }^{26}$ von den Sprecherinnen S. und C. in der Untersuchungszeit nahezu vollständig abgebaut, die Sprecherinnen H. und O. haben diese Variante dagegen in ihrem Sprachgebrauch bis 2009/10 beibehalten (Abb. 2):

$24 \quad$ Das ist meines Erachtens ein zusätzlicher deutlicher Hinweis darauf, dass die Sprecher keine Akkommodation an Gesprächspartner in der konkreten Interaktion vornehmen. Andernfalls wäre die Reduktion im Interview und nicht im Regionalgespräch, denn die regionale Gesprächspartnerin verwendet lokale Formen, wohingegen das Interview auf Hochdeutsch stattfindet.

25 Dasselbe gilt auch für den Gebrauch von neuen Varianten (vgl. dazu ausführlicher Abschnitt 3).

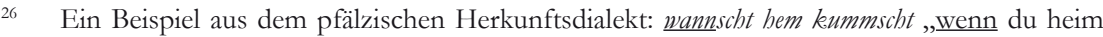
kommst". 


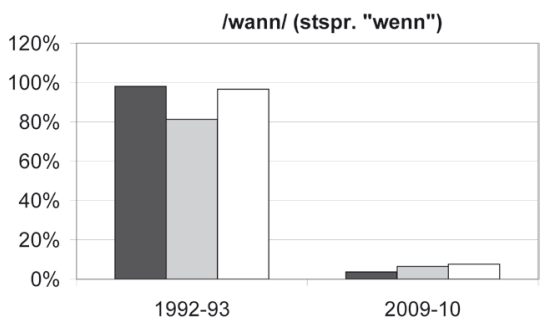

Sprecherin S.

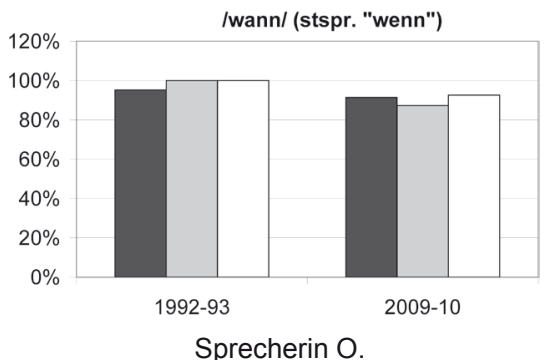

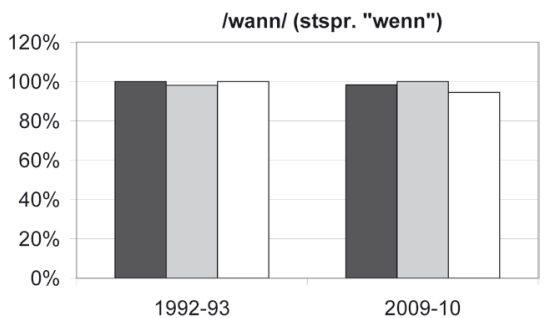

Sprecherin $\mathrm{H}$.

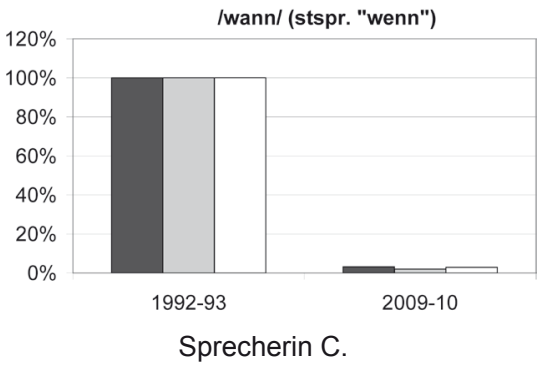

Sprecherin C.

$\square$ ingroup $\square$ outgroup $\square$ Interview

Abb. 2: Entwicklung der Variable wann/wenn in drei Situationstypen bei vier Sprecherinnen in 1992/93-2009/10

Das Beispiel zeigt sehr gut, dass die Abbau- bzw. Erhaltungsprozesse generell sehr konsequent durchgeführt werden. Die Gesamtergebnisse - welche Variablen von den Sprecherinnen bevorzugt beibehalten und welche eher aufgegeben werden - können hier aus Platzgründen nicht vorgeführt werden. Auch kann bisher kein irgendwie plausibler Grund dafür angegeben werden, warum bei Sprechern mit sehr identischen Ausgangsbedingungen ein äußerst unterschiedliches Verhalten gegenüber Erhalt bzw. Abbau von einzelnen Varianten festgestellt werden konnte. Die Frage, ob sich dabei Differenzierungen in primäre und sekundäre Merkmalgruppen (Schirmunski 1931) bzw. Abstufungen wie in einheimischen Dialektregionen (Lenz 2003) ergeben, ist noch zu untersuchen.

\section{Wandel im Varietätenrepertoire: Analyse der Querschnittsdaten}

Im vorherigen Abschnitt wurde der Wandel in real time untersucht. Als wichtigstes Ergebnis konnte die Zunahme von Regionalvarianten in der Gegenwart und das Erscheinen der intrasituativen Variation festgestellt werden. In 
diesem Abschnitt geht es darum, zu untersuchen, ob es situationsbedingte Unterschiede in Bezug auf die zwei externen Situationen (outgroup-Gespräch und Interview) in der aktuellen Sprachgebrauchsphase der Einwanderungsgeneration gibt und welche Veränderungen seit 1992 stattgefunden haben. Daraus sollen Erkenntnisse in Bezug auf das aktuelle Varietätenrepertoire gewonnen werden. Es geht wieder zunächst um die Sprecherin S. AnschlieBend wird ein Vergleich mit anderen Sprechern durchgeführt werden.

\subsection{Intersituativer Vergleich}

Wenn wir noch einmal zu den beiden zuletzt analysierten Fallbeispielen 3 und 4 zurückkehren und die dargestellten Analyseergebnisse miteinander vergleichen, so zeigt sich bei der Sprecherin S. im Ausschnitt „Leintuch“ (Interview) und im Ausschnitt „Silberne Hochzeit“ (outgroup-Gespräch) ein identisches Sprachgebrauchsmuster. Beim Vergleich lassen sich z.B. folgende Gemeinsamkeiten feststellen:

- Merkmale des Herkunftsdialekts in beiden Fallbeispielen,

- Merkmale des lokalen Dialekts der Aufnahmeregion (Saarländisch), und zwar sowohl im Gespräch mit der Saarländerin als auch im Interview mit dem Hochdeutsch sprechenden Interviewer,

- Standard/Dialekt-Variation: hochdeutsche und dialektale Merkmale werden in beiden Datensets nebeneinander und abwechselnd verwendet,

- zwischen- bzw. interdialektale Variation: neue, regionaltypische autochthone Dialektvarianten kommen zu den ,alten, mitgebrachten“ Varianten hinzu und werden in beiden Situationstypen ebenfalls nebeneinander und abwechselnd verwendet.

Die Gemeinsamkeiten werden auch bestätigt durch die quantitative Analyse. Der Dialektanteil beträgt im Gespräch mit der Saarländerin 73,9 und im Interview 69,8 Prozent. Es liegt kein signifikanter Unterschied vor, die Sprecherin zeigt ein sehr identisches Sprachverhalten in Bezug auf alle untersuchten Variablen der Standard/Dialekt-Variation in beiden Situationen.

Tabelle 3 zeigt den Vergleich des Dialektanteils für sieben Variablen in den beiden untersuchten Situationstypen. Hier zeigt sich sehr deutlich, dass in Bezug auf die Vorkommenshäufigkeit der Dialektvarianten keine wesentlichen Unterschiede vorliegen: Der prozentuale Anteil der Dialektvarianten in beiden Situationen ist identisch.

Vergleicht man jedoch die einzelnen Variablen untereinander, so zeigen sich große Unterschiede in Hinblick auf die Verteilung von dialektalen und standardnahen Varianten: Der Dialektanteil von ,nicht ${ }^{6}($ net) z.B. beträgt im Interview 98,6 Prozent, wohingegen der von, wenn' (wann) nur 7,6 Prozent ausmacht. 
Dass die verschiedenen Varianten des Herkunftsdialekts ${ }^{27}$ unterschiedlich stark abgebaut werden, ist nicht überraschend: Das wurde oben bereits diskutiert. Die Sprecherin ist sich der Unterschiede offensichtlich bewusst und baut die verschiedenen Merkmale im Laufe der Zeit zu unterschiedlichem Grad ab. Was jedoch überraschend ist und ein wesentliches Merkmal des Varietätenrepertoires (nicht nur dieser Sprecherin, sondern auch typisch für die anderen Sprecher dieser Migrantengruppe) zu sein scheint, ist die fast fehlende Differenzierung in Bezug auf unterschiedliche Gesprächspartner und die Kommunikationssituation. So beträgt z.B. der Dialektanteil von, nicht' (net) im outgroup-Gespräch 95 Prozent, und der von ,wenn' (wann) ebenfalls nur 6,5 Prozent, d.h. ein nahezu identisches Ergebnis wie im Interview.

\begin{tabular}{|c|c|c|c|c|c|c|}
\hline \multirow{4}{*}{ Variable } & \multicolumn{6}{|c|}{2009} \\
\hline & \multicolumn{3}{|c|}{ Gespräch } & \multicolumn{3}{|c|}{ Interview } \\
\hline & \multirow{2}{*}{$\frac{\sum}{\text { abs. }}$} & \multicolumn{2}{|c|}{ Dialektanteil } & \multirow{2}{*}{$\frac{\sum}{\text { abs. }}$} & \multicolumn{2}{|c|}{ Dialektanteil } \\
\hline & & abs. & $\%$ & & abs. & $\%$ \\
\hline $\begin{array}{l}\text { /st/-/št/ } \\
\text { (Palatalisierung) }\end{array}$ & 142 & 102 & 71,8 & 111 & 74 & 66,7 \\
\hline $\begin{array}{l}\text { /b/-/w/ } \\
\text { (Spirantisierung) }\end{array}$ & 62 & 55 & 88,7 & 63 & 52 & 82,5 \\
\hline $\begin{array}{l}\text { /ei/-/e/ } \\
\text { (Monophthongierung) }\end{array}$ & 162 & 140 & 86,4 & 133 & 118 & 88,7 \\
\hline /-n/-/ø/n-Apokope & 515 & 474 & 92,0 & 469 & 375 & 80,0 \\
\hline nicht - net & 179 & 170 & 95,0 & 143 & 141 & 98,6 \\
\hline wenn - wann & 62 & 4 & 6,5 & 66 & 5 & 7,6 \\
\hline wir - mir & 109 & 105 & 96,3 & 152 & 146 & 96,1 \\
\hline
\end{tabular}

Tab. 3: Übersicht über den Dialektanteil bei 7 ausgewählten Variablen in den Sprachdaten der Sprecherin S. in zwei Situationstypen im Jahr 2009

Das hat sich auch bei der Analyse von neuen regionalen Varianten gezeigt. Bei diesen Varianten des Saarländischen handelt es sich um solche Merkmale, die das Saarländische vom Herkunftsdialekt unterscheiden: das Hilfsverb han 1./3.P.Pl. (im Herkunftsdialekt hen), das Hilfsverb han 1.P.Sg. (im

$27 \quad$ Wie in Anmerkung 17 schon ausgeführt, deckt sich der Herkunftsdialekt in Bezug auf einzelne Merkmale mit dem Saarländischen. Es kann jedoch davon ausgegangen werden, dass das Abbauverhalten der Varianten damit nicht zusammenhängt. Ebenso verhält es sich beim intersituativen Abbau: Der Status der Varianten (russlanddeutsch oder saarländisch) ist dabei anscheinend nicht relevant. Weitere Untersuchungen zu dieser Frage sind allerdings notwendig. 
Herkunftsdialekt hab/haw), das „endungslose“ Partizip (z.B. gefunn, im Herkunftsdialekt gfunne) und das nicht synkopierte Präfix ge- im Partizip Perfekt (gehat, im Herkunftsdialekt ghat).

Es zeigt sich, dass die Probandin sehr spärlich neue Regionalvarianten gebraucht. Eine Ausnahme sind die Formen han (Sg.: ich han „ich habe“; Pl.: mir han „wir haben") und die Diskurspartikel ne (nicht? nicht wahr?). Bemerkenswert ist jedoch - und das bestätigt die oben dargestellte Nicht-Differenzierung der Kommunikationssituation und des konkreten Gesprächspartners -, dass der Anteil von han im standardsprachlichen Interview sogar höher ist als im outgroup-Gespräch (vgl. Abb. 3). Auch wenn dieses Übergewicht Zufall sein kann, so kann nicht übersehen werden, dass die Form han in der Varietät der Probandin ihren festen Platz hat - neben der herkömmlichen Form hab (bzw. haw) und der standardsprachlichen Form ich habe - und dass sie diese Formen als für die externe Kommunikation angebracht betrachtet und verwendet.

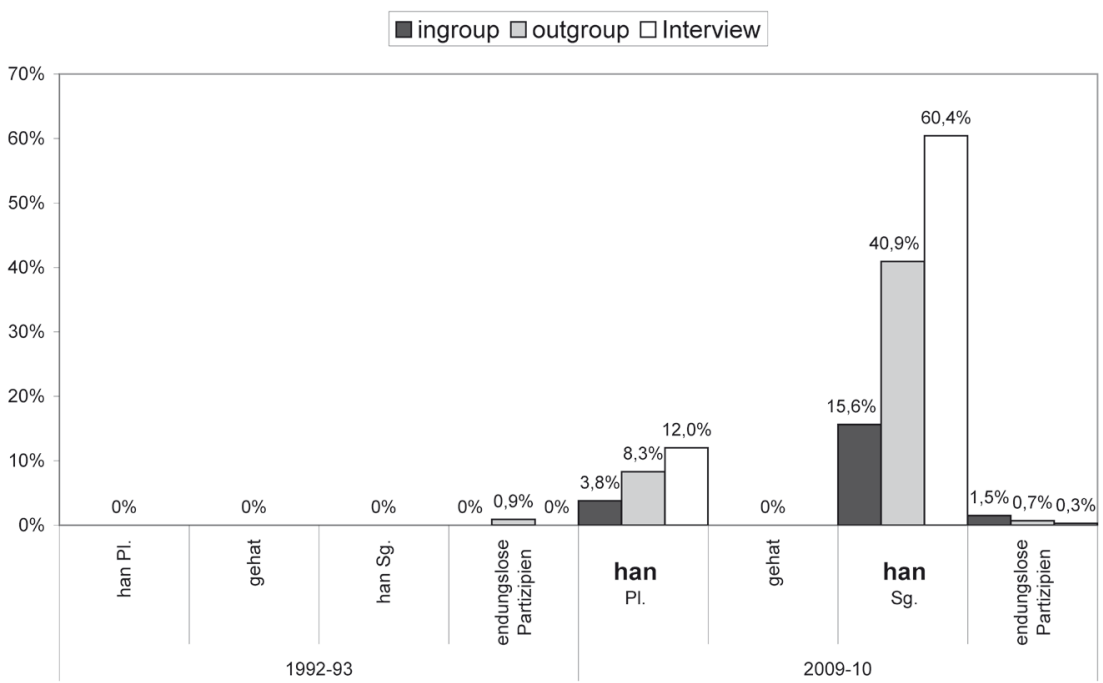

Abb. 3: Anteil der vier saarländischen Varianten im diachronen und synchronen Vergleich in den Daten der Sprecherin S. in drei Situationen (ingroup-Gespräch, outgroup-Gespräch, Interview)

Interessant ist nun jedoch, dass die Probandin das Hilfsverb han auch in einem ingroup-Gespräch mit ihrer Mutter verwendet, das ebenfalls 2009 stattgefunden hat (vgl. Abb. 3, han). Laut Auer/Hinskens (2005) wäre hier die dritte, höchste Stufe der Akkommodation erreicht, wenn der Sprachwandel sich in der gesamten speech community (Migrantengruppe) ausbreitet (vgl. Auer/Hinskens 2005, S. 336). Hier stellt sich mit Chambers jedoch generell die Frage, ob es gerechtfertigt sei, von accommodation zu sprechen, und ob es 
nicht sinnvoller wäre, hier dialect acquisition anzunehmen (Chambers 1992). Denn in der ingroup-Kommunikation stehen die Sprecher nicht unter Anpassungsdruck und der einzige Grund für die Aufgabe von Dialektvarianten könnte der Erwerb von neuen (standardsprachlichen und regional/saarländischen) Varianten sein, die dann die ursprünglichen Varianten nicht nur in der externen Kommunikation, sondern auch in der Kommunikation innerhalb der eigenen Migratengruppe ersetzen. Hier sind noch weitere detaillierte Untersuchungen notwendig.

\subsection{Sprechervergleich}

Der Sprechervergleich wurde in Bezug auf zwei Fragen durchgeführt: 1) das Verhalten der Sprecher zum Abbau bzw. Erhalt von mitgebrachten eigendialektalen Merkmalen, und 2) Akkommodation bzw. Erwerb von neuen lokalen Varianten des Saarländischen. Abbildung 4 zeigt exemplarisch die Ergebnisse des Sprechervergleichs in Bezug auf den Abbau bzw. Erhalt der uns schon bekannten dialekttypischen Variable wann, wenn'. Die Ergebnisse der longitudinalen Analyse in Bezug auf die vier Sprecherinnen wurden bereits dargestellt (vgl. Abb. 2). Nur bei zwei Sprecherinnen liegt ein erheblicher Rückgang des Gebrauchs vor (Sprecherin S. und Sprecherin C.), und zwar durchgehend in allen drei untersuchten Situationen (somit auch in der internen Kommunikation); kein Abbau liegt dagegen bei den Sprecherinnen $\mathrm{O}$. und $\mathrm{H}$. vor.

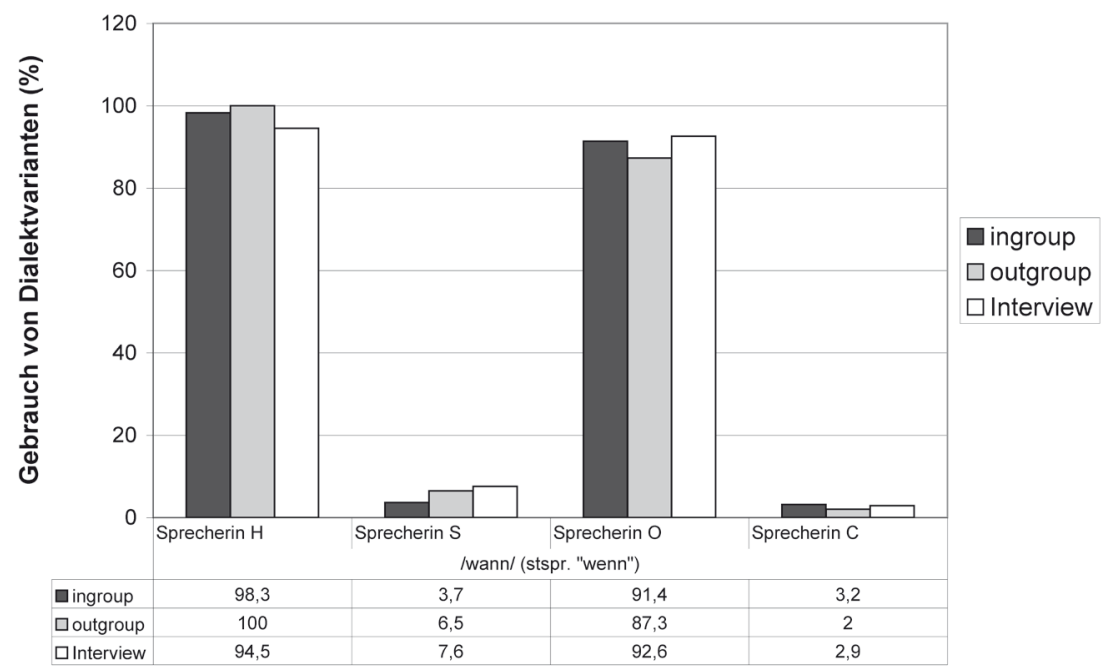

Abb. 4: Übersicht über den Stand des Dialektgebrauchs in 2009/10 (Variable wenn/wann) bei 4 Sprecherinnen 
Es hat sich somit gezeigt, dass die Variationsmuster der oben untersuchten Sprecherin S. und die Konvergenzergebnisse im aktuellen Varietätenrepertoire im Sprechervergleich bestätigt wurden. Es stellte sich bei allen bisher untersuchten Sprechern ein identisch-selektiver Umgang mit dem Abbau bzw. Erhalt von konkreten Variablen heraus. Wenn Varianten abgebaut bzw. beibehalten werden, dann situationsunabhängig, wie bereits für die Sprecherin S. nachgewiesen. Es liegt auch bei anderen Sprechern kaum situationsgebundene adressatenspezifische interpersonelle Akkommodation an den aktuellen Gesprächspartner in der Interaktion vor.

Die Ergebnisse des Sprechervergleichs in Bezug auf die Frage des Erwerbs von neuen saarländischen Varianten sind in zwei Richtungen interessant:

1) Die Sprecherinnen gebrauchen unterschiedliche Varianten des Saarländischen.

2) Die Sprecherinnen gebrauchen dieselben Varianten des Saarländischen sowohl im outgroup-Gespräch als auch im Interview.

Die Abbildungen 5 und 6 stellen die Ergebnisse des Sprechervergleichs in Bezug auf die zweite Frage dar.

Wie bereits oben ausgeführt, ist das Hilfsverb han im Sg. und Pl. die am häufigsten vorkommende Form bei der oben untersuchten Sprecherin S. Die Sprecherin H. verwendet dagegen diese Form nicht. Ihre einzige saarländische Variante ist das westpfälzische endungslose Partizip, und zwar vergleichbar häufig in beiden Situationen (jeweils 19,4 und 22,2 Prozent, vgl. Abb. 5 und 6). Bei der Sprecherin O. kommt die Form han ebenfalls häufig vor, sowohl im outgroup-Gespräch (jeweils 93,2 Prozent im Pl. und 96,1 Prozent im Sg.) als auch im Interview (jeweils 97,9 Prozent im Pl. und 90,7 im Sg.). Der Gebrauch von han ist somit viel frequenter bei dieser Sprecherin im Vergleich zu der Sprecherin S. Die Probandin O. gebraucht zudem neben han auch besonders häufig die typisch saarländische Form gehat. ${ }^{28}$ Diese Form spielt bei den anderen Probanden jedoch kaum eine Rolle. Die Sprecherin C. verwendet im Unterschied zu O. kaum regionalsprachliche Varianten, mit einer kleinen Ausnahme (das endungslose Partizip in beiden Situationen, jedoch jeweils nur 1,2 und 1,3 Prozent).

Wichtig für die aktuelle Fragestellung des Beitrags ist die Feststellung, dass die Sprecherinnen sich offensichtlich für verscbiedene saarländische Varianten „entschieden“ haben, dass aber alle Sprecher ohne Ausnahme keinen Unterschied machen zwischen den verschiedenen outgroup-Situationen. Somit findet keine interpersonelle Akkommodation in der aktuellen Interaktion statt, sondern es wird die gleiche „externe“ Varietät für alle Kommunikationsereignisse außerhalb der ingroup-Kommunikation verwendet.

28 Im Herkunftsdialekt wäre es die Variante ghat (mit e-Synkope). 


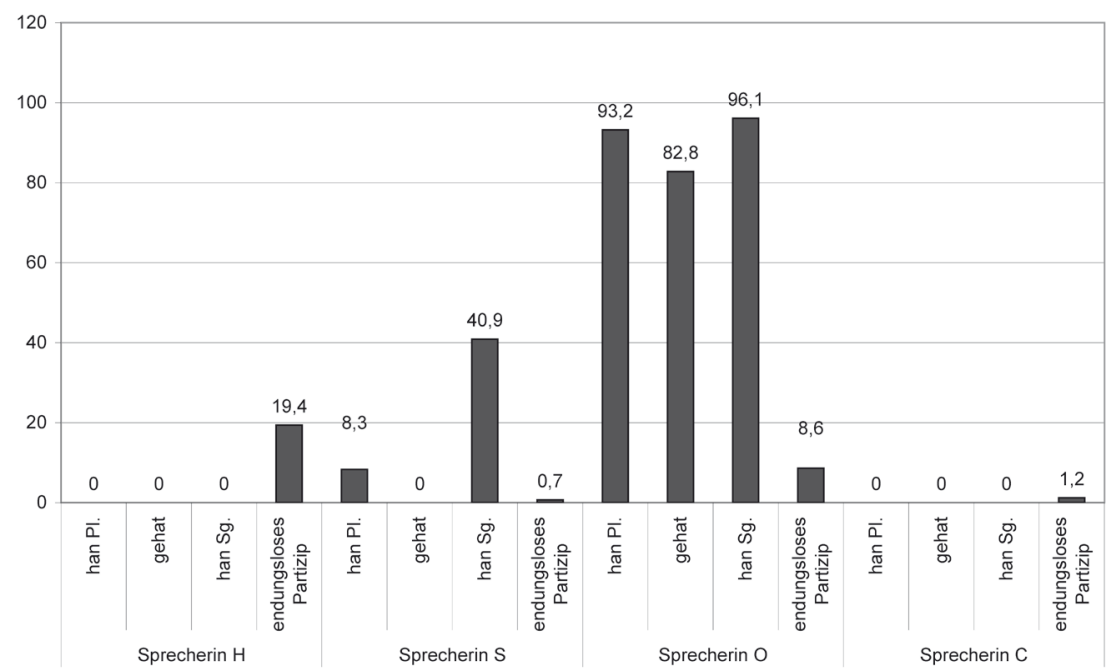

Abb. 5: Anteil der saarländischen Varianten bei 4 Sprecherinnen für vier Variablen 2009/10 im outgroup-Gespräch (\%)

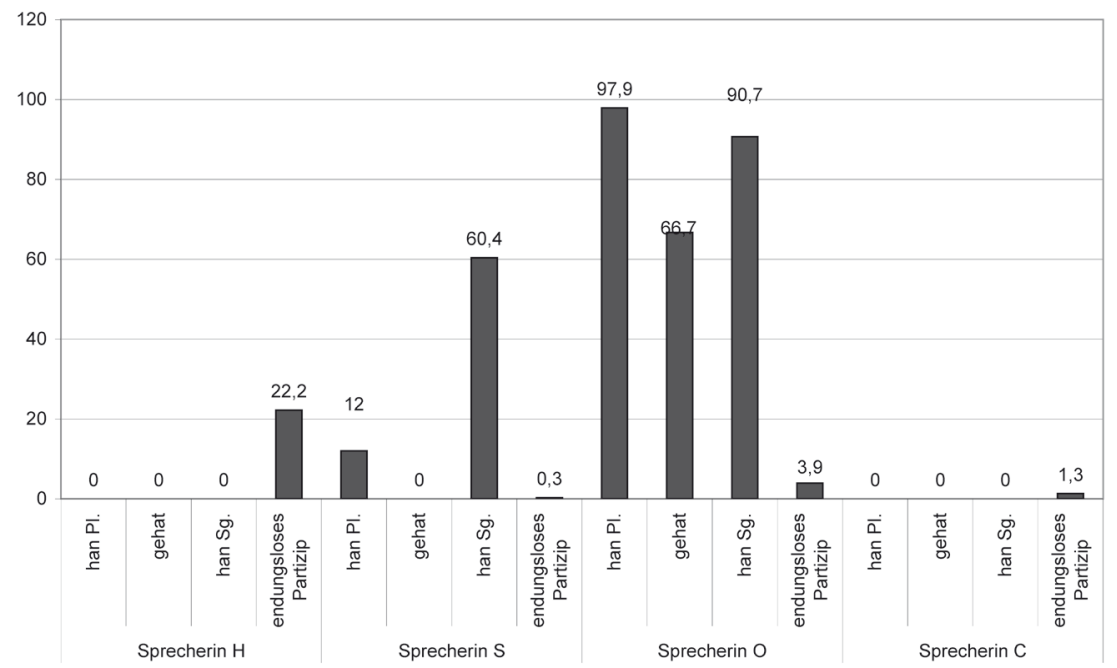

Abb. 6: Anteil der saarländischen Varianten bei 4 Sprecherinnen für vier Variablen in 2009/10 im Interview (\%) 


\section{Diskussion}

Abschließend möchte ich zu den drei Anfangsfragen zurückkehren.

\section{$\mathrm{Zu}$ (i): Ist Spracbwandel in real time festzustellen und wie ist er in die Sprachsituation des Einwanderungslandes ein₹uordnen?}

Wie die Längsschnittanalysen veranschaulichen, handelt es sich bei den Veränderungen vom linguistischen Gesichtspunkt aus um eine gewisse Abnahme der hochdeutschen Varianten in den externen Kommunikationssituationen. Das zeigt sich als grundsätzliche Tendenz bei den meisten Sprechern. Vom soziolinguistischen Gesichtspunkt her bedeutet dies eine gewisse Lockerung, d.h. eine Veränderung der Einstellung zu den außerhalb der eigenen Sprechergruppe zu realisierenden Varietäten. Die Lockerung äußert sich in der Abkehr von der Orientierung auf die Produktion des Hochdeutschen und im Zulassen von Dialektvarianten. Im Laufe des knapp zwei Jahrzehnte andauernden Aufenthalts im bundesdeutschen Sprachgebiet haben die deutschsprachigen Minderheiten einen Wandel in der öffentlich wahrnehmbaren Varietät vollzogen, der als eine Art soziolinguistische Destandardisierung betrachtet werden kann.

Obwohl der Prozess in einigen Punkten anders verläuft als bei einheimischen Sprechern, passt dieser Prozess in Grundzügen in den allgemeinen soziolinguistischen Kontext der Destandardisierung in Deutschland. Denn unter Destandardisierung wird allgemein die „Weiterentwicklung der lebendigen deutschen Sprache mitunter gegen die schriftliche Norm“ (Sperber/ von Polenz 1966, S. 103-105) bzw. die „Liberalisierung der Normen der Standardsprache unter dem Einfluss der Umgangssprachen“" (Guchman in Serebrennikow 1973, S. 484) verstanden. Die Destandardisierung wird als einer der wichtigsten soziolinguistischen Prozesse der Sprachgegenwart gesehen (Auer 2000). ${ }^{29}$

Der allgemeine Unterschied zum Destandardisierungsprozess im bundesdeutschen Kontext besteht u.a. darin, dass bei den dialektsprechenden Zuwanderern nicht nur vertikale Konvergenz auf der Standard/DialektEbene stattfindet. In jedem Konvergenzprozess bei Migrantenvarietäten sind mindestens zwei dialektale Varietäten involviert, d.h. es findet hier auch selektive horizontale Konvergenz zwischen verschiedenen Dialektvarietäten statt: der Herkunftsdialekt und die neue autochthone Varietät der Region. ${ }^{30}$ Das konnte zumindest bei den hier untersuchten Sprecherinnen nachgewiesen werden.

$29 \quad$ Vgl. auch Mattheier/Radtke (Hg.) (1997); allerdings anders: Schmidt/Herrgen (2011).

30 Allerdings können Varianten des neuen Dialekts von Migranten auch als Prestige-Varianten angesehen werden; dann wäre es komplexer, denn es würde sich um eine Art vertikale Konvergenz handeln. In diesem Bereich sind noch weitere Untersuchungen notwendig. 
$\mathrm{Zu}$ (ii): Welche Hinweise geben die durchgefübrten real-time-Analysen auf das aktuelle Sprachrepertoire der Einwanderungsgeneration?

Das Sprachrepertoire der Einwanderungsgeneration hat sich durch die diachronen Wandelprozesse ebenfalls verändert.

Die erste Veränderung betrifft die strukturelle Distanz zwischen den einzelnen Varietäten des Repertoires. Wenn im Anfangsstadium die Varietäten der Aussiedler strukturell streng voneinander getrennt waren, so nähern sie sich mit der zunehmenden Aufenthaltsdauer an, und zwar dadurch, dass Dialektvarianten aus der gruppeninternen Kommunikation in die gruppenexterne übernommen werden, wie oben dargestellt wurde. ${ }^{31}$ Hier stellt sich aktuell eine Ähnlichkeit mit den Prozessen der Herausbildung von regionalen Gebrauchsstandards in Deutschland dar, wenn spezifische, früher nur der internen Kommunikation vorbehaltene Regionalmerkmale Eingang in die öffentliche Sprachvarietät, den sog. „Gebrauchsstandard“ finden. ${ }^{32}$

Zweitens sind die einzelnen Kommunkationsbereiche durch starke intrasituative Variation gekennzeichnet, eine neue Erscheinung, die für den Sprachgebrauch der Russlanddeutschen in den Herkunftssprachinseln und unmittelbar nach der Migration nicht typisch war. Auch hier haben sich die Zuwanderer den Sprachgebrauchsmustern der autochthonen Sprecher angenähert. Sie wechseln zwischen verschiedenen Varianten innerhalb einer Interaktion, ohne dass sich die äußeren (situativen) Bedingungen und der Kommunikationspartner verändern (ausführlich dazu die Diskussion zu (3) unten).

Es gibt jedoch einen Bereich, in dem die Migranten der Einwanderungsgeneration - einfach ausgedrückt - „noch“ nicht den soziolinguistischen Stand der Aufnahmegesellschaft erreicht haben: Für den gruppenexternen Kommunikationsbereich erscheint ihr Varietätenrepertoire im Vergleich zu autochthonen Sprechern als weniger ausdifferenziert. In Bezug auf verschiedene gruppenexterne kommunikative Situationen liegen hier kaum Unterschiede im Sprachgebrauch von Varianten vor und folglich hat im Varietätenrepertoire noch keine intersituative Differenzierung stattgefunden. Die geringen Unterschiede bei der intersituativen Distributionsanalyse belegen, dass die untersuchten Sprecher sprachlich keinen Unterschied zwischen einem nachbarschaftlichen Gespräch oder einem formalen Interview machen.

Die von Auer/Hinskens (2005) diskutierte Hypothese der weitgehenden Irrelevanz der interpersonal accommodation wird somit durch die Analyse der russischsprachigen Migranten der Einwanderungsgeneration grundsätzlich unterstützt. Die Migranten haben eine bestimmte Vorstellung von der „angemessenen outgroup-Varietät", die sie dann unabhängig vom konkreten

31 Die Übernahme von Standardvarianten in die ingroup-Kommunikation wurde im vorliegenden Beitrag nicht diskutiert. Näheres dazu in Berend (2012).

32 Vgl. dazu Auer (2000) und jetzt besonders Deppermann/Kleiner/Knöbl (i.Dr.). 
Gesprächspartner und der Gesprächssituation verwenden. Die Analyseergebnisse lassen die Schlussfolgerung zu, dass in der aktuellen Entwicklungsphase keine adressatenspezifische interpersonelle Akkommodation in der gruppenexternen Interaktion stattfindet. Der Varietätenwandel vollzieht sich durch die Aufnahme bestimmter in der Wahrnehmung der Migrantensprecher als „stereotyp“ (Auer/Hinskens 2005) erscheinender lokaler Merkmale, die dann permanent als externe Gebrauchsvarietät in Einsatz kommt. Dieser Stereotypisierungsprozess scheint bei Migranten stärker ausgeprägt zu sein als bei autochthonen Sprechern und spiegelt die Tatsache wider, dass in der Sprechergemeinschaft der Zugewanderten vor allem der übergeordnete Kontrast zwischen Ingroup- und Outgroup-Kommunikation relevant ist.

\section{$\mathrm{Zu}$ (iii): Bilden sich neue Regionalvarietäten des Deutschen aus?}

In der migrationsbasierten Varietäten- und Stilforschung wird gegenwärtig die Frage diskutiert, ob es sich um Varietäten oder Stile handelt (allerdings in Bezug auf die 2. und 3. Generation). Hinskens (2007) postuliert z.B. die Herausbildung von „new types of non-standard Dutch“ in Bezug auf die Situation in den Niederlanden, und stellt die Frage, ob es sich hier um ,coherent language systems“ bzw. um sich entwickelnde „continuums of intermediate varieties" handelt (ebd., S. 288) und an anderer Stelle fragt er bezüglich dieser neuen Varietäten, ob sie Ethnolekte oder „ethnic styles“ darstellten (Hinskens 2011).

Die hier vorgestellte Analyse bezog sich bisher auf individuelle Repertoires, unter der Annahme, dass es sich um diskrete Varietäten handelt. Diese Annahme basierte ursprünglich, zu Beginn der Datenerhebung 1992, auf der Beobachtung, dass die von russlanddeutschen Zuwanderern produzierten eigenständigen Formen in sich geschlossene Sprachvarietäten bilden (z.B. in der Definition von von Polenz 199933). Nach den oben beschriebenen Prozessen des Wandels stellt sich die grundsätzliche Frage nach dem Status der neuen outgroup-Varietäten dieser Migrantengruppe in Bezug auf die Frage ihrer Regionalität. Es handelt sich um Varietäten in oben vorgestellter Definition; auf der Grundlage der vorgestellten Einzelanalysen ist jedoch unklar, wie diese Varietäten einzuschätzen sind und ob es gewichtige Argumente gibt, diese Varietäten als deutsche Regional- bzw. regionale Umgangssprachen anzusehen. ${ }^{34}$ Es muss die Frage gestellt werden, ob es sich in gewisser Weise um Regionalsprachen als Produkt der Standard/DialektVariation handelt.

33 „[...] eine bestimmte Menge sprachlicher Varianten, die über eine bloße Zufallsmenge hinaus eine außersprachlich determinierte „spezifische Kombination von Varianten“ darstellt" (von Polenz 1999, S. 415).

34 Die Frage der russischen Sprachkontaktphänomene in diesen Varietäten wird hier nicht berücksichtigt. 
Betrachten wir zunächst die Faktoren, die gegen eine Einschätzung als „Regionalvarietät“ des Deutschen sprechen. Die Gegenargumente betreffen vor allem die Variationstypen und die Strukturierung der Variation. Die Verwendung von standardsprachlichen und regionalen Formen im deutschsprachigen Raum ist bestimmten Regeln unterlegen. So führt z.B. Möller (2006) aus, dass zwischen Dialekt und Standardsprache „durchaus nicht alles möglich ist [...], dass sich Kookkurenzrestriktionen feststellen lassen, die eine - auch historisch plausible - klare Trennung zwischen dialektalem und nichtdialektalem Substandard nahelegen“ (ebd., S. 101). In jeder Dialektregion existieren eigene Kookurrenzrestriktionen. So nennen z.B. Kallmeyer/ Keim (1994) sechs Stufen bei den „Mischvarianten“ für Mannheim und zeigen somit die entsprechende Komplexität:

Für die dialektale Lage sind Formen belegt wie /uffgschriewe/ und /uffgschriwwe/ [...] , d.h. die Variante 5.2.2 kann auch vor der Variante 4 auftreten. Nicht belegt dagegen sind Formen wie */uffgschrieben/ oder*/uffgeschriebe/. Die Stufe 3 und 4 sind nicht austauschbar, */aufgschriewen/ ist nicht belegt. (ebd., S. 154)

Genau solche Formen wie uffgeschriebe bzw. aufgschriewen jedoch, die aufgrund von Kookkurenzrestriktionen in Mannheim nicht vorkommen, sind ein typisches Merkmal der Strukturierung der Variation in den untersuchten Migrantenvarietäten. Wie in den Analysen gezeigt wurde, stimmen nämlich die Kookkurenzrestriktionen nicht mit denen in einheimischen Varietäten (z.B. in der Darstellung von Kallmeyer/Keim oben für das Pfälzische) bzw. es liegen keine bzw. andere Kookkurrenzrestriktionen vor.

Auch die Strukturierung der Variation nach dem Muster des CodeShifting, d.h. dem ,allmählichen Gleiten auf dem Standard/Dialekt-Kontinuum“" (Auer 1986, S. 119), ist im analysierten Material anders. Beim Migrantendeutsch gibt es kein koordiniertes, auf den Standard- oder Dialektpol orientiertes Shifting. Das führt ebenfalls dazu, dass - anders als im bundesdeutschen Kontext - der intrasituativen Variation im Allgemeinen seltener bzw. weniger deutliche konversationelle Funktionen zugeschrieben werden können. Es gibt häufig keine Indizien für die sozial-symbolische Funktion der Variation wie sie in einzelnen Untersuchungen beschrieben wurde. ${ }^{36}$

Trotz der Evidenz dieser Faktoren sollen einige Argumente angefüht werden, die das Migrantendeutsch den Regionalsprachen ähnlich machen.

So ist in Bezug auf die Strukturiertheit und Systematik der Variation anzumerken, dass es auch im deutschen Sprachraum Variationstypen gibt, die keinen systematischen Regeln unterliegen. Man spricht dann z.B. von Fluktuation. Dazu merkt Auer (1986, S. 119) an, dass ,einzelne Parameter - in verschiedene Richtung - ohne (für Teilnehmer und Wissenschaftler)

\footnotetext{
35 Nach Kallmeyer/Keim (1994, S. 147-152), bedeutet Stufe 3: Verdunkelung, Rückverlegung und Vertiefung bzw. Aufhellung, Vorverlegung und Anhebung von Vokalen; Stufe 4: Kürzung bzw. Längung von Vokalen: widda (wieder), hawwe (haben), kuche (Kuchen); Variante 5.2.2: uff.

36 Vgl. Kallmeyer/Keim (1994) und jetzt besonders Knöbl (2012).
} 
erkennbare Systematik“ variieren. Das erinnert sehr an die Strukturierung der Migrantenvarietät und ist dem in der Sprachkontaktforschung in jüngster Zeit häufig beschriebenen mixing ähnlich. ${ }^{37}$

Ein weiteres Argument betrifft die der Variation zugeschriebenen Funktionen, eine Frage, die in der Varietäten- und Sprachkontaktforschung häufig diskutiert wird. Bezüglich der Funktionen ist man sich in der aktuellen Forschung nicht unbedingt einig. Denn auch im bundesdeutschen Kontext sind Beispiele beschrieben worden, bei denen - ähnlich wie bei Migranten - keine konversationelle Funktion für den Gebrauch der einen oder anderen Variante festzumachen ist, insbesondere bei dem eben erwähnten mixing, das im Gegensatz zum switching eher keine Funktionen haben soll (vgl. Auer 1999; siehe auch Knöbl (2012) zur Analyse des Standard-Dialekt-Mixing bei jungen Sprechern).

Macha (1991) beschreibt den funktionslosen Wechsel als „buntes Nebenund Durcheinander heterogener Varietätenanteile“ bzw. als „sprachliche Gemengenlage":

[...] Es wechseln dialektale mit standardsprachlichen Syntagmen und Sätzen; in hochsprachliche Redeketten werden plötzlich Dialektismen eingebaut und umgekehrt. Dabei ist auf Anhieb schwer auszumachen, ob das Wechselverhalten „spontan“ oder ,konditioniert" ist. (ebd., S. 210)

Es scheint also so zu sein, dass die für das Migrantendeutsch übliche Strategie der ,motivlosen Variation' auch im deutschen Sprachraum sogar häufig der Fall ist und dass auch einheimische Dialektsprecher häufig Varianten mischen, ohne dafür offensichtliche Gründe zu haben.

Die Ausführungen machen deutlich, dass es für die zuletzt gestellte Frage, ob sich neue Regionalvarietäten des Deutschen herausbilden, noch keine eindeutige Antwort gibt. Die von den Migranten der Einwanderungsgeneration verwendeten Varietäten können meines Erachtens am besten als „migrationsbasierte“ Regionalsprachen des Deutschen bezeichnet werden. ${ }^{38}$

\section{Transkription}

Die Transkripte sind lautnah auf der Basis der orthographischen Zeichen dargestellt. Folgende Gesprächsmerkmale wurden berücksichtigt:

$\begin{array}{ll}\uparrow \downarrow & \text { steigende bzw. fallende Intonation } \\ = & \text { Verschleifung/Elision (z.B. mi=di mit den) } \\ {[]} & \text { Überlappungen und Simultansprechen } \\ \text { ne/ } & \text { Wortabbruch (net , ,nicht }{ }^{\circ} \text { ) }\end{array}$

37 Auer beschreibt mixing am Beispiel der bilingualen Variation, schließt es aber auch nicht für die Standard/Dialekt-Variation aus.

38 In den analysierten Fallbeispielen ging es um pfälzisch ausgerichtete Migrationsvarietäten. Bairische, schwäbische und weitere Migrationsvarietäten dieser Art sind denkbar. 
$() /.(-) /(--) \quad$ kürzere und längere Pausen

(...) unverständlich

[...] Auslassung

((lacht) $\quad$ interpretierende Kommentare

\section{Literatur}

Auer, Peter (1986): Konversationelle Standard/Dialekt-Kontinua (Code-Shifting). In: Deutsche Sprache 14, S. 97-124.

Auer, Peter (1999): From code-switching via language mixing to fused lects. Toward a dynamik typology of bilingual speech. In: International Journal of Bilingualism 3, S. 309-332.

Auer, Peter (2000): Processes of horizontal and vertical convergence in present day Germany. In: Målbryting 4, S. 9-26.

Auer, Peter (2007): Mobility, contact and accommodation. In: Llamas, Carmen/Mullany, Louise/Stockwell, Peter (Hg.): The routledge companion to sociolinguistics. Routledge, S. 109-115.

Auer, Peter/Hinskens, Frans (2005): The role of interpersonal accommodation in a theory of language change. In: Auer, Peter/Hinskens, Frans/Kerswill, Paul (Hg.): Dialect change: convergence and divergence in European languages. Cambridge, S. 335-357.

Barden, Birgit/Großkopf, Beate (1998): Sprachliche Akkommodation und soziale Integration: Sächsische Übersiedler und Übersiedlerinnen im rhein-moselfränkischen und alemannischen Sprachraum. (= Phonai 43). Tübingen.

Bellmann, Günter/Herrgen, Joachim/Schmidt, Jürgen Erich (2002): Mittelrheinischer Sprachatlas. Bd. 5: Morphologie. Tübingen.

Berend, Nina (1998): Sprachliche Anpassung. Eine soziolinguistisch-dialektologische Untersuchung zum Rußlanddeutschen. (= Studien zur Deutschen Sprache 14). Tübingen.

Berend, Nina (2005): Regionale Gebrauchsstandards - Gibt es sie und wie kann man sie beschreiben? In: Eichinger, Ludwig M./Kallmeyer, Werner (Hg.): Standardvariation. Wie viel Variation verträgt die deutsche Sprache? (= Jahrbuch des Instituts für Deutsche Sprache 2004). Berlin/New York, S. 143-170.

Berend, Nina (2011): Sprache nach der Re-Migration am Beispiel der russischsprachigen Zuwanderung in Deutschland. In: Thüne, Eva-Maria/Betten, Anne (Hg.): Sprache und Migration. Linguistische Fallstudien. (= Lavori interculturali sul Tedesco 4). Rom, S. 89-111.

Berend, Nina (2012): Migration und Dialektwandel. Aspekte der jüngsten Sprachgeschichte des Russlanddeutschen. In: Bär, Jochen A./Müller, Marcus (Hg.): Geschichte der Sprache - Sprache der Geschichte. Probleme und Perspektiven der historischen Sprachwissenschaft des Deutschen. Oskar Reichmann zum 75. Geburtstag. (= Lingua Historica Germanica 3). Berlin, S. 609-628. 
Berend, Nina (i.Vorb.): Varietätenwandel nach der Migration. Eine Longitudinalstudie zum Sprachgebrauch der Einwanderungsgeneration der Russlanddeutschen in Deutschland.

Berend, Nina/Jedig, Hugo (1991): Deutsche Mundarten in der Sowjetunion. Geschichte der Forschung und Bibliographie. (= Schriftenreihe der Kommission für Ostdeutsche Volkskunde in der Deutschen Gesellschaft für Volkskunde 53). Marburg.

Chambers, Jack (1992): Dialect acquisition. In: Language 68, S. 673-705.

Clyne, Michael/Eisikovits, Edina/Tollfree, Laura (2002): Ethnolects as in-group varieties. In: Duszak, Anna (Hg.): Us and others. Social identities across languages, discourses and cultures. Amsterdam, S. 133-157.

Deppermann, Arnulf/Kleiner, Stefan/Knöbl, Ralf (i.Dr.): ,Standard usage‘: Towards a realistic conception of spoken standard German. In: Auer, Peter/Reina, Javier Caro/Kaufmann, Göz (Hg.): Language variation - European perspectives IV: Selected papers from the 6th International Conference on Language Variation in Europe (ICLaVE 6), Freiburg, June 2011. (= Studies in Language Variation). Amsterdam/Philadelphia.

Diener, Irina (2004): Muttersprache - Lernerfahrungen einer russlanddeutschen Mutter und Lehrerin in Deutschland. In: Deutsch als Zweitsprache 2004, S. 21-25.

Häcki Buhofer, Annelies/Burger, Harald (1998): Wie deutschschweizer Kinder Hochdeutsch lernen. Der ungesteuerte Erwerb des gesprochenen Hochdeutschen durch deutschschweizer Kinder zwischen sechs und acht Jahren. (= Zeitschrift für Dialektologie und Linguistik - Beihefte 98). Stuttgart.

Hinskens, Frans (2007): New types of non-standard Dutch. In: Fandrych, Christian/ Salverda, Reinier (Hg.): Standard, variation and language change in Germanic languages. (= Studien zur Deutschen Sprache 41). Tübingen, S. 281-300.

Hinskens, Frans (2011): Emerging Moroccan and Turkish varieties of Dutch: Ethnolects or ethnic styles? In: Kern, Friederike/Selting, Margret (Hg.): Ethnic styles of speaking in European metropolitan areas. (= Studies in Language Variation 8). Amsterdam/Philadelphia, S. 101-129.

Jedig, Hugo H. (1970): Nižnenemeckij govor Altajskogo kraja, tom I (fonetika i morfologija), tom II (sintaksis). Doktorskaja dissertacija. Kafedra nemeckogo jazyka Omskogo gosudarstvennogo pedagogičeskogo instituta imeni A. M. Gorkogo. Omsk, 1970.

Kallmeyer, Werner/Keim, Inken (1994): Phonologische Variation als Mittel der Symbolisierung der sozialen Identität in der Filsbachwelt. In: Kallmeyer, Werner (Hg.): Kommunikation in der Stadt. Teil 1: Exemplarische Analysen des Sprachverhaltens in Mannheim. (= Schriften des Instituts für Deutsche Sprache 4.1). Berlin.

Keim, Inken (1982): Gastarbeiterdeutsch als Spiegel der Kontaktprozesse. In: Ureland, Per Sture (Hg.): Die Leistung der Strataforschung und der Kreolistik. Typologische Aspekte der Sprachkontakte. Akten des 5. Symposions über Sprachkontakt in Europa, Mannheim 1982. (= Linguistische Arbeiten 125). Tübingen, S. 433-445.

Kerswill, Paul (1994): Dialects converging. Rural speech in urban Norway. (= Oxford Studies in Language Contact). Oxford. 
Kleiner, Stefan et al. (2011): „Deutsch heute“. Ein sprachgebietsweites Forschungsprojekt zur regionalen Variation in der gesprochenen deutschen Standardsprache. In: Pohl, Heinz-Dieter (Hg.): Akten der 10. Arbeitstagung für bayerisch-österreichische Dialektologie in Klagenfurt 2007. (= Klagenfurter Beiträge zur Sprachwissenschaft, Jg. 34-36 (2008-2010)). Wien, S. 179-193.

Kloss, Heinz (1976): Abstandsprachen und Ausbausprachen. In: Göschel, Joachim/ Nail, Norbert/Van der Elst, Gaston (Hg.): Zur Theorie des Dialekts. Aufsätze aus 100 Jahren Forschung mit biographischen Anmerkungen zu den Autoren. (= Zeitschrift für Dialektologie und Linguistik - Beihefte 16). Wiesbaden, S. 301-322.

Knipf-Komlósi, Elisabeth (2011): Wandel im Wortschatz der Minderheitensprache. Am Beispiel des Deutschen in Ungarn. (= Zeitschrift für Dialektologie und Linguistik - Beihefte 145). Stuttgart.

Knöbl, Ralf (2012): Dialekt - Standard - Variation. Formen und Funktionen von Sprachvariation in einer mittelschwäbischen Schulklasse. (= OraLingua 1). Heidelberg.

Lenz, Alexandra (2003): Struktur und Dynamik des Substandards: eine Studie zum Westmitteldeutschen (Wittlich/Eifel). (= Zeitschrift für Dialektologie und Linguistik - Beihefte 125). Stuttgart.

Macha, Jürgen (1991): Der flexible Sprecher: Untersuchungen zu Sprache und Sprachbewußtsein rheinischer Handwerksmeister. (= Veröffentlichung des Instituts für Geschichtliche Landeskunde der Rheinlande der Universität Bonn). Köln.

Mattheier, Klaus/Radtke, Edgar (Hg.) (1997): Standardisierung und Destandardisierung europäischer Nationalsprachen. (= VarioLingua 1). Frankfurt a.M.

Möller, Robert (2006): Mögliches und Unmögliches zwischen Dialekt und Standard. Kookkurrenzrestriktionen als Zugang zur Struktur regionaler Umgangssprache im Rheinland. In: Gessinger, Joachim/Voeste, Anja (Hg.): Dialekt im Wandel. Perspektiven einer neuen Dialektologie. (= Osnabrücker Beiträge zur Sprachtheorie 71). Bremen, S. 101-117.

Ramge, Hans (1982): Dialektwandel im mittleren Saarland. (= Veröffentlichungen des Instituts für Landeskunde im Saarland 30). Saarbrücken.

Schirmunski, Viktor (1931): Sprachgeschichte und Siedlungsmundarten I-II. In: Germanisch-romanische Monatsschrift 18, 3-4, S. 112-122; 5-6, S. 171-188.

Schmidt, Jürgen Erich/Herrgen, Joachim (2011): Sprachdynamik. Eine Einführung in die moderne Regionalsprachenforschung. (= Grundlagen der Germanistik 49). Berlin.

Serebrennikow, B.A. (1973): Allgemeine Sprachwissenschaft. Bd.1: Existenzformen, Funktionen und Geschichte der Sprache. Von einem Autorenkollektiv unter der Leitung von B.A. Serebrennikow. München.

Sperber, Hans/von Polenz, Peter (1966): Geschichte der deutschen Sprache. 5., neubearb. Aufl. (= Sammlung Göschen 915). Berlin.

Trudgill, Peter (1983): On dialect: social and geographical perspectives. Oxford.

Trudgill, Peter (1986): Dialects in contact. (= Language in Society 10). Oxford.

von Polenz, Peter (1999): Deutsche Sprachgeschichte vom Spätmittelalter bis zur Gegenwart. Bd. 3: 19. und 20. Jahrhundert. Berlin/New York. 